ARTICLE

\title{
Arabidopsis RBV is a conserved WD40 repeat protein that promotes microRNA biogenesis and ARGONAUTE1 loading
}

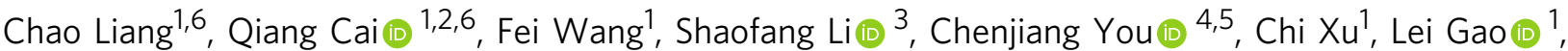

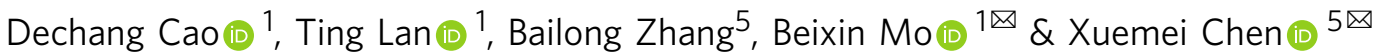

MicroRNAs (miRNAs) play crucial roles in gene expression regulation through RNA cleavage or translation repression. Here, we report the identification of an evolutionarily conserved WD40 domain protein as a player in miRNA biogenesis in Arabidopsis thaliana. A mutation in the REDUCTION IN BLEACHED VEIN AREA (RBV) gene encoding a WD40 domain protein led to the suppression of leaf bleaching caused by an artificial miRNA; the mutation also led to a global reduction in the accumulation of endogenous miRNAs. The nuclear protein RBV promotes the transcription of MIR genes into pri-miRNAs by enhancing the occupancy of RNA polymerase II (Pol II) at MIR gene promoters. RBV also promotes the loading of miRNAs into AG01. In addition, RNA-seq revealed a global splicing defect in the mutant. Thus, this evolutionarily conserved, nuclear WD40 domain protein acts in miRNA biogenesis and RNA splicing.

\footnotetext{
${ }^{1}$ Guangdong Provincial Key Laboratory for Plant Epigenetics, College of Life Sciences and Oceanography, Shenzhen University, Shenzhen 518060, China.

${ }^{2}$ College of Horticulture, College of Life Sciences, Haixia Institute of Science and Technology, Fujian Agriculture and Forestry University, Fuzhou 350002, China. ${ }^{3}$ College of Biological Sciences, China Agricultural University, Beijing 100193, China. ${ }^{4}$ State Key Laboratory of Genetic Engineering and Collaborative Innovation Center of Genetics and Development, Institute of Plant Biology, School of Life Sciences, Fudan University, Shanghai 200438, China. ${ }^{5}$ Department of Botany and Plant Sciences, Institute for Integrative Genome Biology, University of California, Riverside, CA 92521, USA. ${ }^{6}$ These authors contributed equally: Chao Liang, Qiang Cai. 凶email: bmo@szu.edu.cn; xuemei.chen@ucr.edu
} 
M icroRNAs (miRNAs), 20-24 nucleotides (nt) in length, are one class of endogenous noncoding small RNAs in eukaryotes. miRNAs are players in gene regulatory networks involved in many biological processes such as development, metabolism, and immunity in plants ${ }^{1}$. Target gene expression is regulated by miRNAs post-transcriptionally through RNA cleavage or translation repression ${ }^{2}$.

The biogenesis of plant miRNAs entails a series of steps. Genes encoding miRNAs $(M I R)$ are transcribed into pri-miRNAs that form imperfect stem-loop structures by DNA-dependent RNA polymerase II $(\mathrm{Pol} \mathrm{II})^{3,4}$. MIR transcription is facilitated by Mediator $^{5}$, (NOT2) ${ }^{6}$, CELL DIVISION CYCLE5 (CDC5) ${ }^{7}$, Elongator ${ }^{8}$, the TREX-2 component THP $1^{9}$, and the chromatin remodeling factor $\mathrm{CHR} 2{ }^{10}$. Pri-miRNAs are processed into premiRNAs by DICER-LIKE1 (DCL1) $)^{1,11-13}$. In humans, primiRNAs are thought to be co-transcriptionally processed, as they are associated with chromatin during transcription and processed at sites of transcription before splicing ${ }^{14,15}$. It is proposed that the retention of pri-miRNAs at transcription sites may enhance processing ${ }^{15}$. In Arabidopsis, NOT2, CDC5, and Elongator interact with both Pol II and DCL1 ${ }^{6-8}$, suggesting that primiRNA transcription and processing may be also coordinated in plants. CHR2 probably also acts co-transcriptionally to repress the processing of pri-miRNAs ${ }^{10}$.

The efficient processing of pri-miRNAs requires the doublestranded RNA-binding protein HYPONASTIC LEAVES1 (HYL1) $^{16,17}$ and the zinc finger protein SERRATE (SE) ${ }^{18,19}$, which form the microprocessor complex with DCL1 ${ }^{20,21}$. The three proteins as well as pri-miRNAs are also found in nuclear foci called dicing-bodies (D-bodies) ${ }^{22}$. Pre-miRNAs are processed to miRNA/miRNA* duplexes, which undergo $3^{\prime}$ methylation by the methyltransferase HEN1 to maintain miRNA stability ${ }^{23}$. Finally, the mature miRNA strands associate with ARGONAUTE1 (AGO1) to form RNA-induced silencing complexes (RISCs), which are active forms of miRNAs ${ }^{24-26}$. In Arabidopsis, size exclusion chromatography showed that the molecular weight of miRISCs is similar to that of AGO1, suggesting that miRISCs are bi-molecular AGO1-miRNA complexes, although larger complexes containing AGO1 can also be detected $24,27,28$.

Here, we isolated a mutant with global defects in miRNA biogenesis in Arabidopsis. The mutation is in a previously uncharacterized gene encoding a protein containing seven WD40 repeats, which we designate as $R B V$. $R B V$ promotes the transcription of $M I R$ genes, as loss of function of $R B V$ reduces $M I R$ promoter activity and the occupancy of Pol II at MIR promoters. In addition, $R B V$ promotes the localization of HYL1 in D-bodies. On the basis of these results, we propose that RBV may act to coordinate MIR transcription and pri-miRNA processing in plant miRNA biogenesis. Moreover, the association of miRNAs with AGO1 was drastically decreased and AGO1 resided in complexes larger than miRISCs in the $r b v-1$ mutant, suggesting that RBV promotes the loading of miRNAs into AGO1. $R B V$ also has a global role in pre-mRNA splicing, affecting a set of short introns.

\section{Results}

Isolation of a mutant with defects in miRNA biogenesis. We performed an ethylmethane sulfonate mutagenesis screen for Arabidopsis mutants in miRNA biogenesis, utilizing the veincentered leaf beaching phenotype caused by the phloem-specific expression of an artificial miRNA (amiR-SUL) targeting the SULFUR (SUL) gene as a visible marker for miRNA activity ${ }^{29}$. Several mutants with reduced leaf bleaching were isolated and found to be in genes with known roles in miRNA biogenesis/ activity, such as hyl1-11, dcl1-30, hen1-11, and ago1-25, suggesting that the genetic screen was effective (Supplementary
Fig. 1a, b). The ago1-25 allele isolated in our study was thus named because it harbored the same mutation as the one previously reported ${ }^{30}$. In addition, we isolated a new suppressor mutant with reduced leaf bleaching (Fig. 1a, b); the mutation was designated $r b v-1$ as it was later shown to be in a previously uncharacterized gene that we named REDUCTION IN BLEACHED VEIN AREA (RBV). This mutant exhibited pleiotropic developmental phenotypes, such as reduced root length, smaller plant size, narrow leaves, short stature, increased branching, and reduced fertility (Fig. 1c and Supplementary Fig. 2). Northern blot analyses showed that both amiR-SUL and endogenous miRNAs (miR156, miR159, miR164, miR165, miR167, miR319, and miR390) were moderately reduced in abundance in 14-day-old amiR-SUL rbv-1 seedlings as compared to amiR-SUL seedings (Fig. 1d). We also performed small RNA sequencing with 14-day-old amiR-SUL and amiR-SUL rbv-1 seedlings. Clustering analysis showed that the three biological replicates for each genotype were highly reproducible (Supplementary Fig. 3a). Reads corresponding to miRNAs were normalized against total mapped reads and miRNAs with RPM (reads per million mapped reads) $>10$ in either genotype (average of three replicates) were included in our analyses (Supplementary Data 1). An overall reduction in miRNA abundance in the mutant relative to the amiR-SUL line was observed (Fig. le and Supplementary Fig. 3b), while there were some miRNAs such as miR845a and miR845b showing increased accumulation in the amiRSUL rbv-1 mutant. We also performed RT-qPCR to examine the expression of some miRNAs' target genes. The transcript levels of SPL3 and SPL10 (targets of miR156), PHB, REV, and PHV (targets of miR165/6), MYB33 and MYB65 (targets of miR159), ARF8 (a target of miR167), and CUC2 (a target of miR164) were de-repressed in the amiR-SUL rbv-1 mutant (Fig. 1f).

$R B V$ encodes an evolutionarily conserved WD40 domain protein. The amiR-SUL rbv-1 mutant was backcrossed with the parental amiR-SUL line. In a total of 614 F2 plants, 141 (23\%) exhibited the mutant phenotypes, which is consistent with the phenotype being caused by a single, nuclear, and recessive mutation $\left(\chi^{2}=1.357 ; P=0.244\right.$; (Supplementary Table 1$)$ ). In order to identify the causal mutation in amiR-SUL $r b v-1$, pooled DNA from mutant plants in the F2 of the amiR-SUL rbv-1 $\mathrm{x}$ amiR-SUL cross was used for whole-genome re-sequencing. The results revealed that the amiR-SUL $r b v-1$ phenotype was linked to a single nucleotide change (G-to-A) in the first exon of AT5G64730, causing the change of the encoded amino acid from glycine to glutamic acid (see Methods; Fig. 2a).

In order to confirm that $R B V$ is indeed AT5G64730, a construct of $R B V$ (AT5G64730)-eYFP driven by its native promoter was generated and introduced into amiR-SUL $r b v-1$. Phenotypes of the transgenic plants showed that $p R B V: R B V-e Y F P$ fully rescued the morphological defects of amiR-SUL $r b v-1$ (Fig. 2c). Furthermore, the transgene restored amiR-SUL accumulation as well as leaf bleaching in amiR-SUL $r b v-1$ (Fig. 2d). While the expression of the amiR-SUL target gene, SUL, was increased in the mutant at the RNA and protein levels, the transgene also restored SUL RNA (Fig. 2e) and SUL protein (Fig. 2f) in amiR-SUL $r b v$-1 to wild-type levels. Moreover, the transgene rescued the defects in miR159 and miR319 accumulation in the amiR-SUL rbv-1 mutant (Fig. 2d). Therefore, the miRNA biogenesis and morphological defects of amiR-SUL rbv-1 were attributable to the mutation in $R B V$ (AT5G64730).

$R B V$ encodes a previously uncharacterized protein with seven WD40 repeats (Fig. 2a). In plants, WD40 repeat proteins are numerous, interact with diverse proteins, and act in a 
a

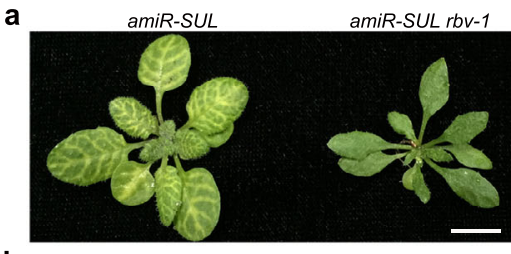

b

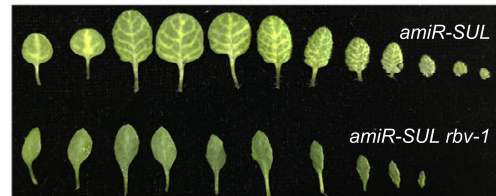

C
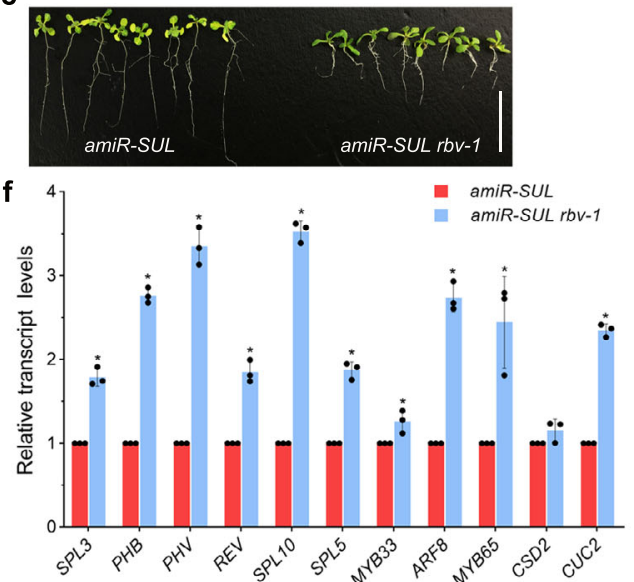

miR156 d

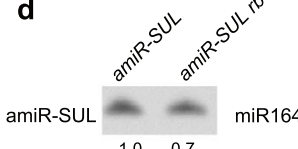

miR164 $=m$ miR159
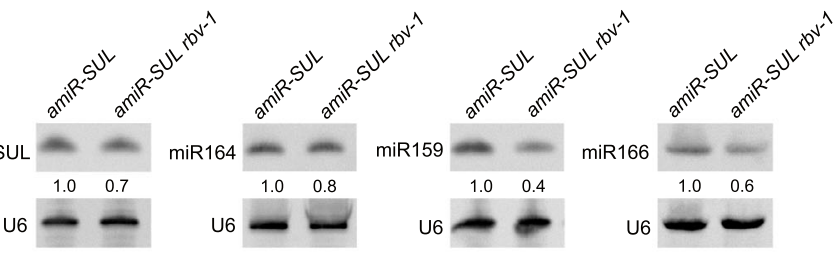

$\begin{array}{ll}1.0 & 0.8\end{array}$

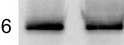

U6

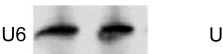

U6

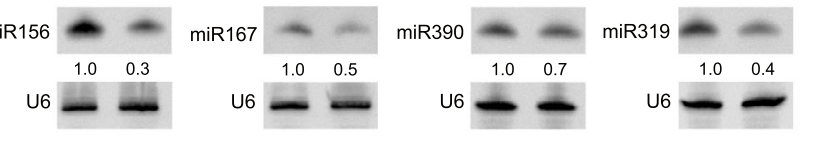

e

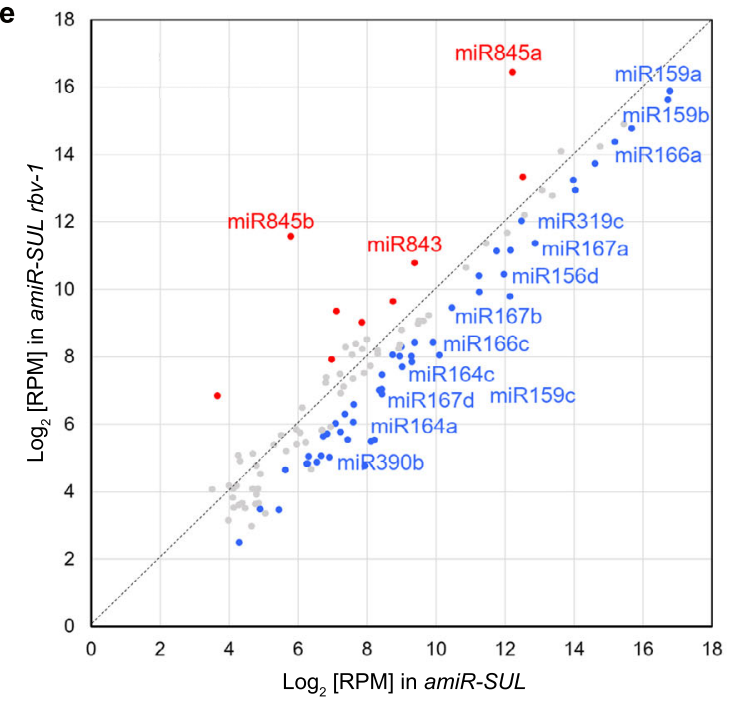

Fig. 1 Isolation of the silencing suppressor mutant rbv-1 from an amiR-SUL line. a Phenotypes of 1-month-old amiR-SUL rbv-1 and amiR-SUL plants. $\mathrm{Bar}=1 \mathrm{~cm}$. b Images of rosette leaves from 1-month-old plants grown under long-day conditions. c 14-day-old seedlings showing reduced root length in amiR-SUL rbv-1. Bar $=1 \mathrm{~cm}$. d RNA gel analysis showing reduced accumulation of amiR-SUL and endogenous miRNAs in the amiR-SUL rbv-1 mutant. U6 was used as an internal control. The numbers represent relative abundance. Two independent repeats gave similar results. e $A$ scatter plot showing the abundance of miRNAs in amiR-SUL rbv-1 and amiR-SUL as determined by small RNA-seq with 14-day-old seedlings. miRNA abundance was calculated as reads per million mapped reads (RPM) and miRNAs with RPM $>10$ in either genotype are shown. The red dots indicate miRNAs with higher levels in amiRSUL rbv-1, and the blue dots indicate miRNAs with lower levels in amiR-SUL $r b v-1$. (Student's $t$ test, ${ }^{\star} P<0.05$ ). $\mathbf{f}$ Determination of miRNA target mRNA levels in amiR-SUL and amiR-SUL rbv-1 in 14-day-old seedlings by RT-qPCR. UBQUITIN5 (UBQ5) was used as the internal control. The values were relative to those in amiR-SUL. Error bars represent standard deviation from three technical replicates. Asterisks indicate a significant difference between amiR-SUL and amiR-SUL rbv-1 (Student's $t$ test, $\left.{ }^{\star} P<0.05\right)$. Source data are provided as a Source Data file.

variety of biological processes, such as plant development and immunity $^{31,32}$. To investigate whether RBV is an evolutionarily conserved protein, a phylogenetic tree in different plants was generated including Angiosperms, Gymnosperms, Bryophytes, Lycophytes, Chlorophyte, and Charophyte algae. In general, Arabidopsis $R B V$ and its nearest paralog (AT5G50230) were separated in the latest common ancestor of multicellular algae (Fig. 2b). A detailed phylogenetic tree and the accession numbers of the proteins used can be found in Supplementary Data 2 and Supplementary Fig. 4a. According to the phylogenetic analysis, $R B V$ orthologs can be found in plants ranging from single-cell green algae to core eudicots and grasses, and there is no close paralog of $R B V$ in the latest common ancestor of land plants. In most eudicots that have undergone gamma whole-genome duplication (WGD) and Brassicaceae that have also undergone beta and alpha WGDs, $R B V$ remained as a single copy. Only in species that have undergone recent, specific WGDs, such as apple, soybean, and maize, there are multiple copies of $R B V$ (Supplementary Fig. 4a). This result indicated that $R B V$ maybe functionally conserved and extra copies might be deleterious.

We also obtained two lines with T-DNA insertions in or near $R B V$ (Salk_126634 and Salk_075672) (Supplementary Fig. 5a, c). Interestingly, the phenotypes of the T-DNA mutants were the same as wild type (Supplementary Fig. 5b). Next, we examined $R B V$ transcripts in the two T-DNA insertion lines. RT-PCR showed that transcripts corresponding to the full-length coding region of $R B V$ were present in the two mutants (Supplementary Fig. 5d). In addition, real-time RT-PCR showed that the levels of $R B V$ transcripts were only mildly reduced in the two mutants (Supplementary Fig. 5e), consistent with the lack of morphological phenotypes.

In order to remove the amiR-SUL transgene background, we crossed the amiR-SUL $r b v$-1 mutant with wild-type (Col) plants. In the F2 population, we identified homozygous rbv-1 plants without the amiR-SUL transgene through genotyping; these plants showed the same pleiotropic phenotypes as amiR-SUL rbv-1 (Fig. 3a). The morphological phenotypes of $r b v-1$ were completely rescued by the $p R B V: R B V$-eYFP transgene (Fig. $3 \mathrm{a})$ ). We further confirmed that the $r b v-1$ mutant without the amiR-SUL transgene had defects in miRNA accumulation. RNA gel blots showed that the levels of several endogenous miRNAs, such as miR156, miR159, miR164 and miR167, were reduced in abundance in $r b v-1$ as compared to wild-type plants. Other miRNAs, such as miR319 and miR398, were unaffected (Supplementary Fig. 6a). To rule out the possibility that RBV indirectly regulated miRNA accumulation by affecting the expression of the miRNA biogenesis machinery, we examined 
a

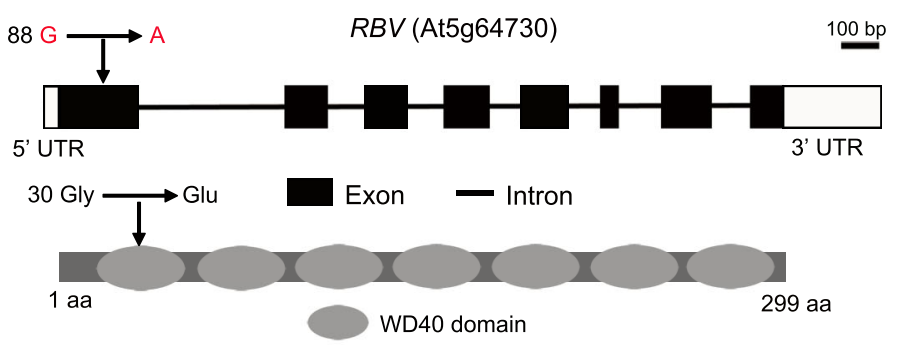

b
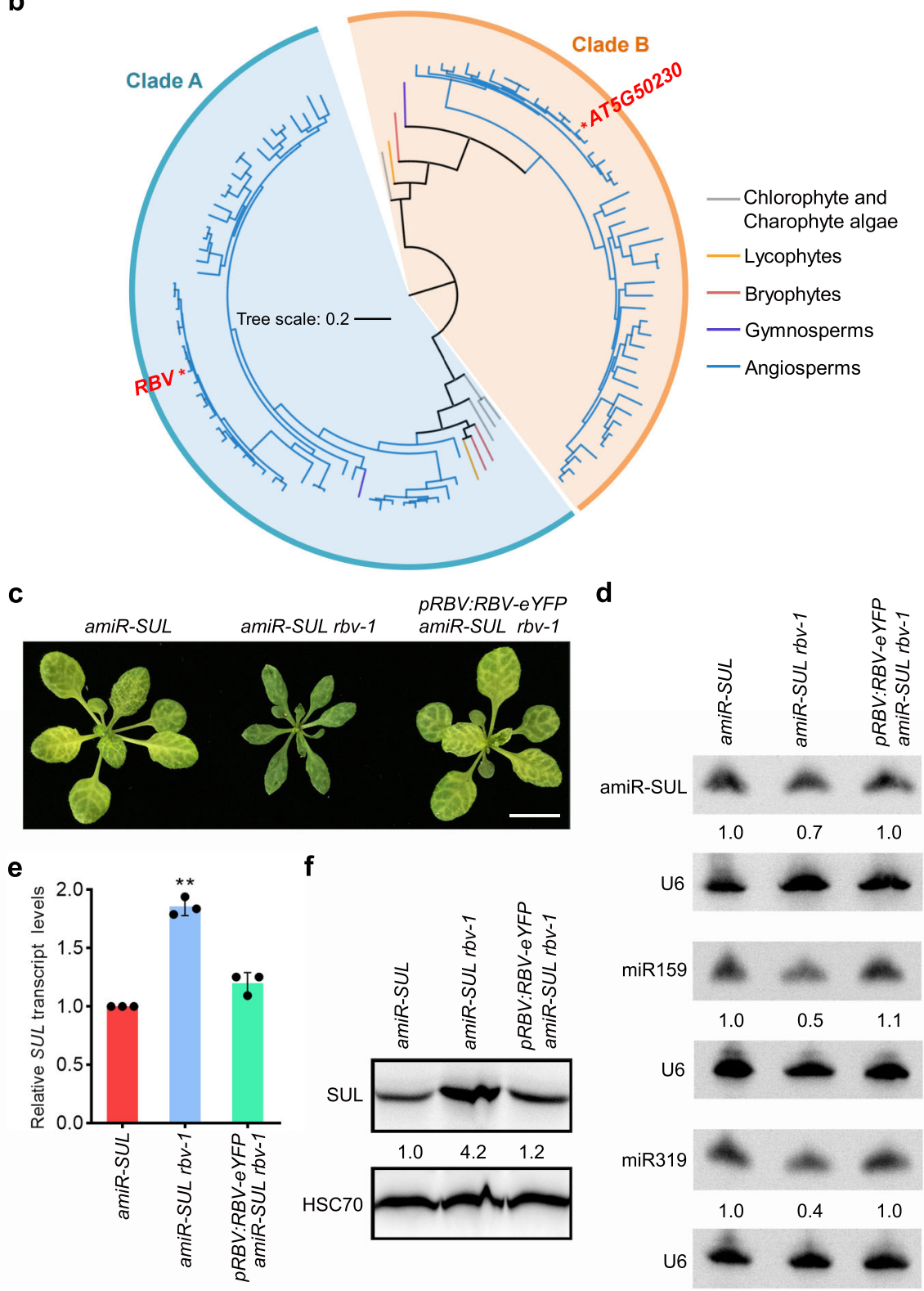

f

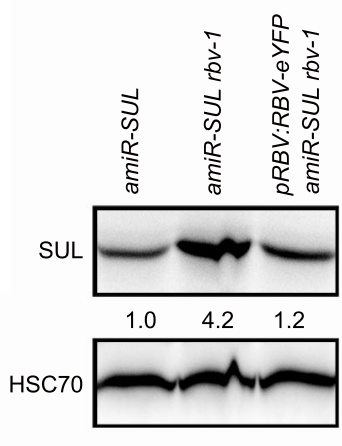

the expression of the known genes involved in miRNA biogenesis, and no significant changes were observed in $r b v$ - 1 (Supplementary Fig. 6b, c).

$R B V$ promotes the transcription of $M I R$ genes. We next investigated how $R B V$ promotes miRNA biogenesis. MIR gene transcription to produce pri-miRNAs is the first step in miRNA biogenesis. We first sought to determine pri-miRNA levels by RNA-seq in Col and $r b v$-1, each with three replicates. Of the 298
pri-miRNAs annotated in Araport11, only 41 gave reads in any sample. Pri-miRNAs with RPKM $>10$ in either $r b v$ - 1 or Col was used for differential expression analysis. Six pri-miRNAs were significantly altered in $r b v$-1 relative to Col with four being decreased and two increased in abundance with $P<0.05$ and foldchange $>1.5$ as the cutoff (Supplementary Fig. 7). As many primiRNAs appeared to be at lower levels in the $r b v-1$ mutant although they did not pass the cutoff (Supplementary Fig. 7), we performed RT-qPCR to determine the levels of seven pri-miRNAs 
Fig. 2 Identification of AT5G64730 as RBV. a Diagrams of the RBV (AT5G64730) gene (upper panel) and protein (lower panel). Rectangles and lines represent exons and introns, respectively. Black and white rectangles represent the coding region and the UTRs, respectively. The point mutation in rbv- 1 and the corresponding change at the amino acid level are indicated (arrows). The protein domains were predicted (http://smart.embl-heidelberg.de/). b A phylogenetic tree of RBV and its paralog At5g50230 in plants. The colors of the branches represent different lineages of plant species. All genes used in the analysis are listed in Supplementary Data 2. The detailed phylogenetic tree is shown in Supplementary Fig. 4a. c Three-week-old plants of the indicated genotypes. pRBV:RBV-eYFP was introduced into amiR-SUL rbv-1. Bar $=1 \mathrm{~cm}$. d RNA gel blot analysis of miRNAs from amiR-SUL, amiR-SUL rbv-1, and the complementation line pRBV:RBV-eYFP amiR-SUL rbv-1 using 14-day-old seedlings. U6 was used as an internal control. The numbers represent relative abundance. e RT-qPCR to determine RNA levels of the amiR-SUL target gene SUL in the indicated genotypes. Three independent biological replicates were used for the calculation of standard deviation. (two-tailed Student's $t$ test, ${ }^{\star \star} P<0.01$ ). $\mathbf{f}$ Protein gel blot analysis to determine the protein levels of the amiRSUL target gene SUL in the indicated genotypes. Two independent repeats gave similar results. Source data are provided as a Source Data file.

a

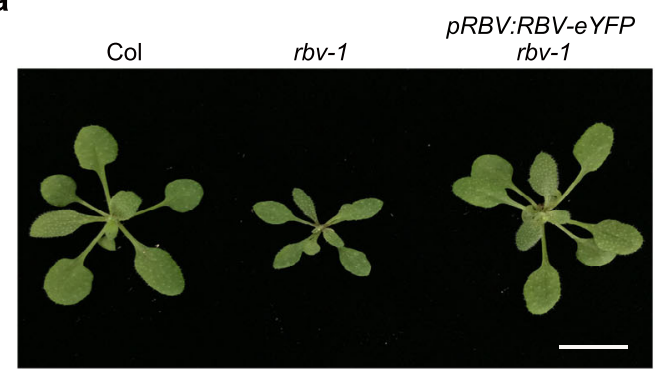

b

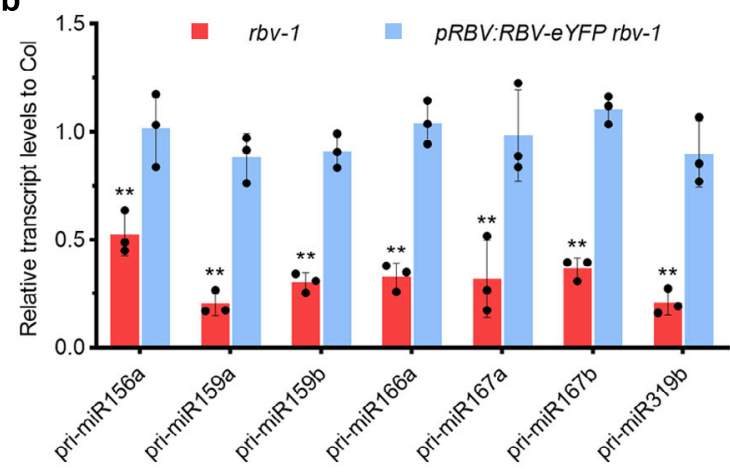

d e

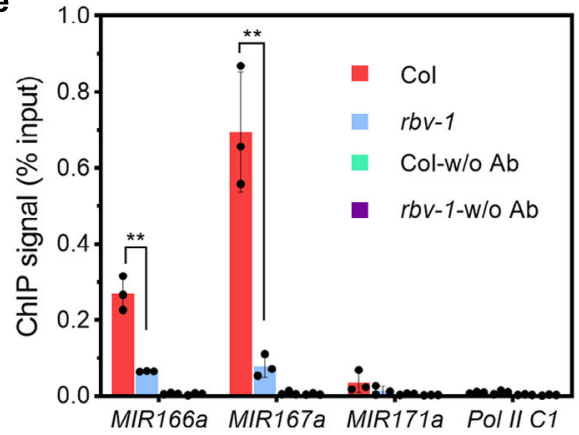

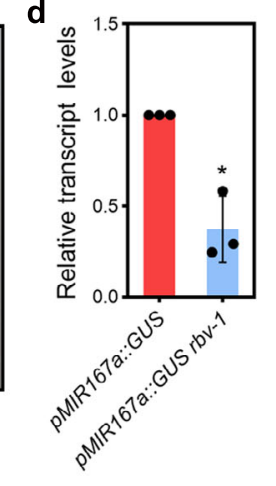

C

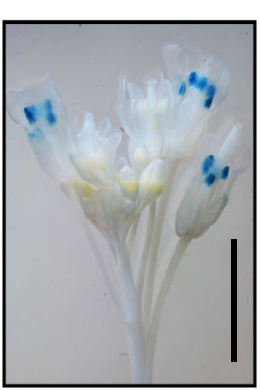

pMIR167a:GUS

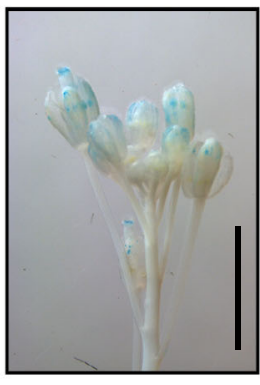

pMIR167a:GUS rbv-1

Fig. 3 RBV promotes the transcription of MIR genes. a Three-week-old plants of the indicated genotypes. Bar $=1 \mathrm{~cm}$. $\mathbf{b}$ Levels of seven pri-miRNAs in 14 day-old seedlings of $r b v-1$ and the complementation line $p R B V: R B V$-eYFP rbv-1 as determined by RT-qPCR. UBQ5 was used as the internal control. Error bars represent standard deviation calculated from three independent replicates. (Student's $t$ test, ${ }^{\star \star} P<0.01$ ). c Representative images of GUS staining of pMIR167a:GUS and pMIR167a:GUS rbv-1 inflorescences. Bars $=2 \mathrm{~mm}$. d Transcript levels of GUS from the two genotypes as determined by RT-qPCR. The expression values were relative to $P M I R 167 a: G U S$. Error bars represent standard deviation calculated from three independent replicates. (two-tailed Student's $t$ test, $\left.{ }^{*} P<0.05\right)$. e RBV is required for the recruitment of Pol II to MIR166a and MIR167a promoters. The occupancy of Pol II at various regions was determined by ChIP with rbv-1 and Col using an antibody that recognizes the C-terminal repeat (YSPTSPS) of the largest subunit of Pol II. ChIP performed without the antibody served as a negative control. A genomic region between the genes AT2G17460 and AT2G17470 named Pol II C1 was also used as a negative control. Mean and standard deviation from three independent replicates are presented. (Student's $t$ test, ${ }^{\star \star} P<0.01$ ). Source data are provided as a Source Data file.

in Col, $r b v-1$ and $p R B V: R B V$-eYFP $r b v-1$. The levels of these primiRNAs were reduced to $\sim 30-50 \%$ of wild-type levels in the $r b v-1$ mutant and the reduction was rescued by $p R B V: R B V-e Y F P$ (Fig. $3 b$ ). The reduction in pri-miRNA abundance could be attributed to impaired MIR genes transcription, reduced stability of pri-miRNAs or enhanced pri-miRNA processing. To determine whether transcription was affected in the $r b v$ - 1 mutant, we crossed $r b v-1$ with a GUS reporter line ( $p M I R 167 a: G U S)$ under the control of the MIR167a promoter and obtained PMIR167a:GUS rbv-1 (with both the transgene and the mutation being homozygous). GUS activity was visibly lower in pMIR167a:GUS $r b v-1$ than in pMIR167a:GUS as revealed by GUS staining (Fig. 3c). RT-qPCR analysis confirmed that the $r b v-1$ mutant had lower GUS transcript levels (Fig. 3d). Thus, reduced transcription of $M I R$ genes could be one of the problems in miRNA biogenesis in $r b v-1$.

To further confirm a positive role of $R B V$ in $M I R$ gene transcription, the occupancy of Pol II at MIR loci was determined by chromatin immunoprecipitation (ChIP) with an antibody against the Pol II C-terminal repeats. ChIP without an antibody served as the negative control. MIR166a, MIR167a, and MIR171a promoter regions, as well as $\mathrm{C} 1$ (a region between the genes AT2G17460 and AT2G17470 known to not engage Pol II ${ }^{7}$ ), were examined by RT-qPCR after ChIP. The MIR166a and MIR167a promoter regions were enriched in the immunoprecipitates in both $r b v-1$ and Col relative to $\mathrm{C} 1$ (Fig. 3e). Pol II occupancy at MIR166a and MIR167a promoter regions was reduced in the $r b v-1$ mutant 
a pRBV:RBV-eYFP pSE:SE-mRuby3
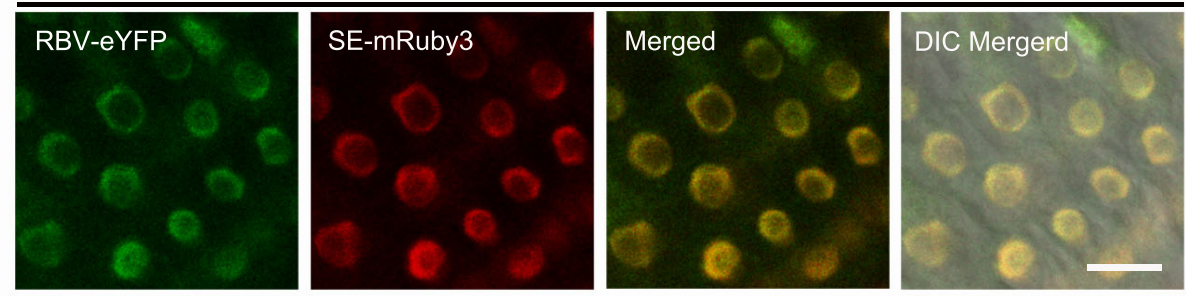

b

pHYL1:HYL1-GFP pSE:SE-mRuby3

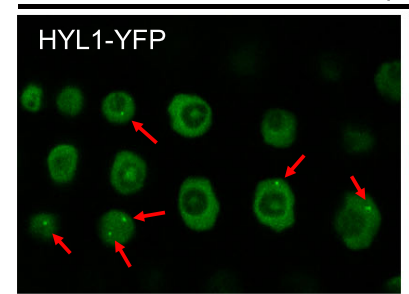

C
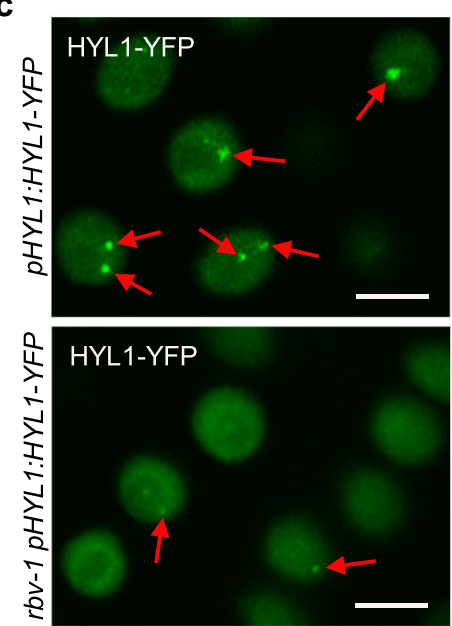
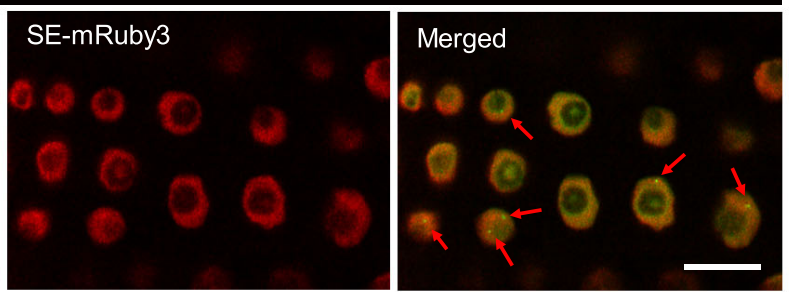

d

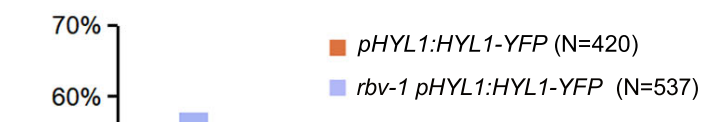

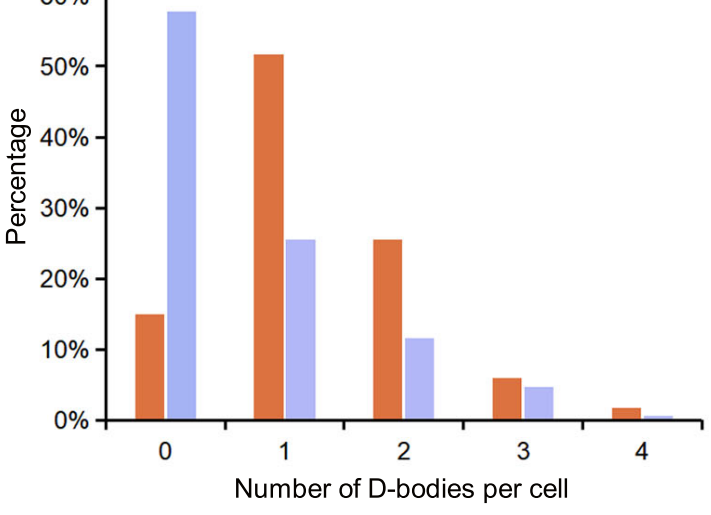

Fig. 4 RBV is required for the proper localization of HYL1 in D-bodies. a RBV is localized in the nucleoplasm. eYFP and mRuby3 signals were detected in root cells $(n=100)$ from pRBV:RBV-eYFP pSE:SE-mRuby3 transgenic plants. Bar $=10 \mu \mathrm{m}$. $\mathbf{b}$ HYL1 and SE protein localization in roots of $p H Y L 1: H Y L 1-Y F P$ pSE:SE-mRuby3 plants. Both proteins show nucleoplasmic localization while HYL1 also shows D-body localization. In total 100 cells were observed. Bar $=10 \mu \mathrm{m}$. c Representative images of $p H Y L 1: H Y L 1$-YFP signals in root cells from the meristematic zone in the two genotypes. Arrows indicate D-bodies. Bars $=5 \mu \mathrm{m}$. $\mathbf{d}$ The percentage of cells with 1-4 D-bodies per cell in wild type and rbv-1. The $x$-axis represents the number of D-bodies per cell, and the $y$ axis represents the percentage of cells with the corresponding number of D-bodies. " $N$ " means the numbers of total root cells examined. Source data are provided as a Source Data file.

relative to Col, while the signal on MIR171a was too low to be calculated (Fig. 3e). Thus, $R B V$ facilitates the recruitment of Pol II to $M I R$ loci.

$\mathrm{RBV}$ is localized in the nucleoplasm and required for the proper localization of HYL1 in D-bodies. We studied the expression of $R B V$ in various tissues and the subcellular localization of the protein. RT-PCR analyses of RNAs from seedlings, roots, cauline leaves, rosette leaves, stems, and inflorescences showed ubiquitous $R B V$ expression (Supplementary Fig. 8a). $R B V: R B V$-eYFP transgenic plants exhibited YFP fluorescence in the nucleoplasm but not the nucleolus (Fig. 4a). SE is a component of the microprocessor in pri-miRNA processing and a mRuby3-tagged SE protein driven by the SE promoter was also localized in the nucleoplasm, as previously reported ${ }^{9}$ (Fig. 4a). The nucleoplasmic localization of the two proteins prompted us to ask whether RBV interacts with SE. However, results of yeast two-hybrid, BiFC, and co-IP experiments did not show interactions between the two proteins (Supplementary Fig. 8c-e).

HYL1 and DCL1 form nuclear foci known as D-bodies, which are sites of pri-miRNA processing ${ }^{22,33}$. To determine the status of D-bodies in the $r b v-1$ mutant, we crossed a $p H Y L 1: H Y L 1-Y F P$ transgene ${ }^{22}$ into the mutant and obtained plants homozygous for both the transgene and the $r b v-1$ mutation. D-body numbers were determined in 420 and 537 root nuclei of wild-type and $r b v-1$ plants, respectively. The number of HYL1-YFP D-bodies was significantly decreased in $r b v-1$ (Fig. 4c, d), suggesting that mutation of RBV leads to a defect in D-body formation or the localization of HYL1 to D-bodies. Although SE was reported to form D-bodies in N. benthamiana ${ }^{22}$ and Arabidopsis ${ }^{33}$, we have never observed SE D-bodies or nuclear speckles in Arabidopsis roots, no matter when SE was fused to mRuby or eCFP (Fig. 4a and Supplementary Fig. 8b). Even upon co-expression of $p S E: S E-$ mRuby3 and pHYL1:HYL1-YFP, SE D-bodies cannot be observed despite obvious HYL1 D-bodies (Fig. 4b). 
a

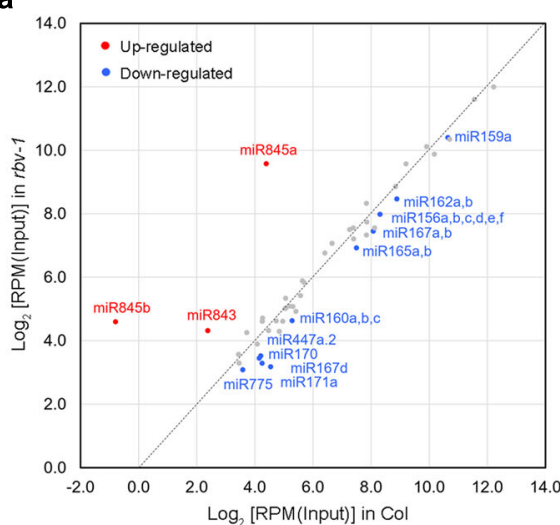

b

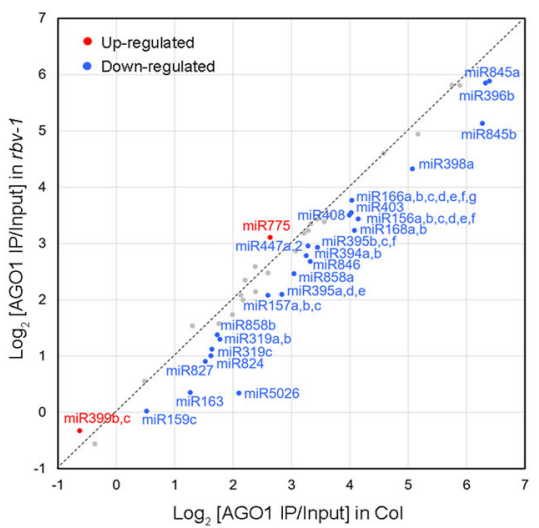

C

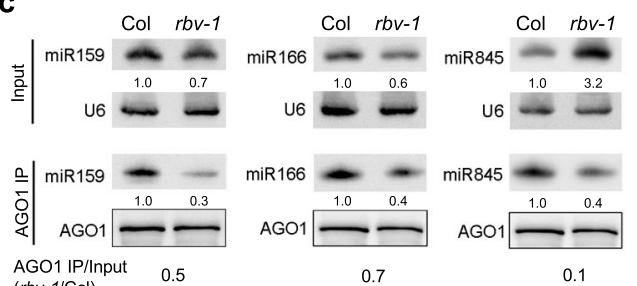
$\underset{(r b v-1 / C o l)}{\mathrm{A}}) \quad 0.5$

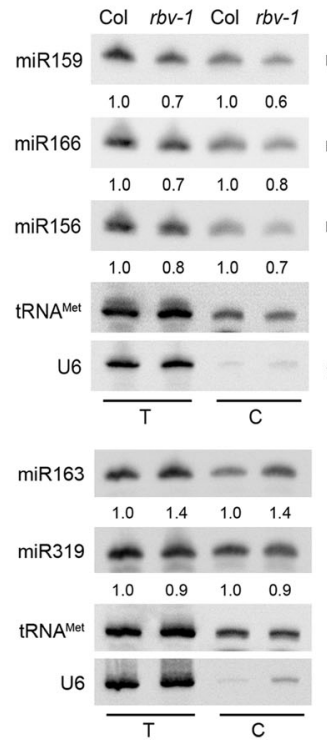

d

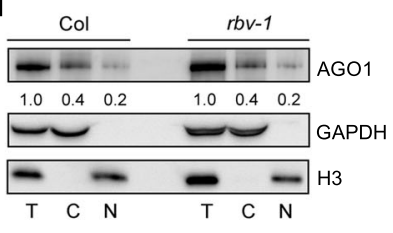

$\mathbf{f}$
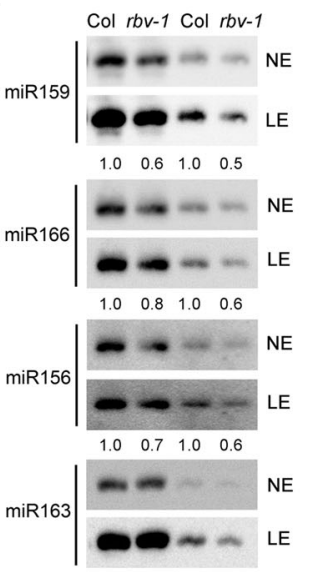

$\begin{array}{llll}1.0 & 1.0 & 1.0 & 0.5\end{array}$

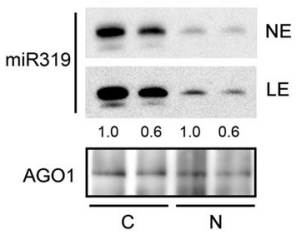

g

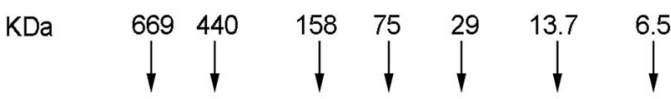

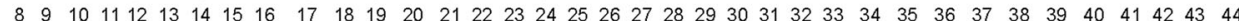
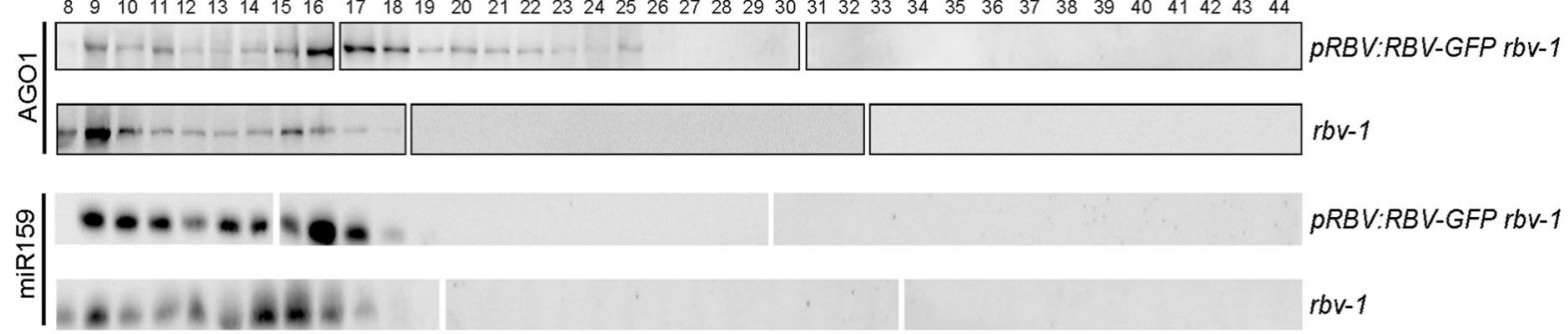

Mutation of $R B V$ leads to a defect of miRNA loading into AGO1. miRNAs are loaded into AGO1 to form miRISCs that execute the silencing of target RNAs ${ }^{24,26,34}$. To determine whether $R B V$ affects the formation of miRISCs, we performed AGO1 IP followed by sRNA-seq with both input and IP samples from wildtype and $r b v-1$ seedlings. Three replicates were performed and showed high reproducibility (Supplementary Fig. 9). From input samples, many miRNAs showed a small but statistically significant reduction in abundance in the $r b v-1$ mutant (Supplementary Data 3). A few miRNAs, such as miR163, miR845a, miR845b and miR843, were elevated in abundance in the mutant (Fig. 5a; Supplementary Data 3). The loading status of each miRNA was expressed as the ratio of miRNA abundance between AGO1 IP and input and differences between wild type and $r b v$ - 1 were evaluated by student's $t$-test. A global reduction in the AGO1 loading of miRNAs was observed in the $r b v-1$ mutant (Fig. 5b; Supplementary Data 4). Notably, miR845a and miR845b, which exhibited elevated levels, were less associated with AGO1 in the mutant, indicating that RBV is crucial for miRISC formation. RNA gel blots were also performed to validate the sRNA-seq results. miR159 and miR166 levels were lower in input and further reduced in AGO1 IP in the mutant (Fig. 5c). miR845a levels were strongly increased in the $r b v-1$ input sample as compared with Col input but greatly reduced in the $r b v-1$ AGO1 IP sample as compared with Col AGO1 IP (Fig. 5c; Supplementary Data 4). Thus, the $r b v-1$ mutant exhibits a global miRNA loading defect.

AGO1 is a nuclear-cytoplasmic shuttling protein and is thought to load miRNAs in the nucleus ${ }^{25}$. We performed nuclearcytoplasmic fractionation to determine whether RBV affects the nuclear-cytoplasmic partitioning of AGO1. The results showed that the cytoplasmic/nuclear $(\mathrm{C} / \mathrm{N})$ ratios of $\mathrm{AGO} 1$ were similar between $\mathrm{Col}$ and the $r b v-1$ mutant (Fig. 5d). We also detected miRNAs by gel blots in cytoplasmic and nuclear fractions. The results showed that miR159, miR166, miR156, and miR319 were similarly decreased in the nuclear and cytoplasmic fractions in $r b v$ 1 (Fig. 5e). Thus, RBV did not affect the nuclear-cytoplasmic partitioning of AGO1 or miRNAs. Next, we performed AGO1 IP followed by northern blotting to determine the miRNA loading efficiency. Using the ratio of miRNA abundance in AGO1 IP vs. input to represent miRNA loading efficiency, the calculated 
Fig. 5 Mutation of RBV leads to a defect of miRNA loading into AGO1. a A scatter plot of miRNA abundance in rbv-1 input vs. Col input. All miRNAs were normalized by total reads, and those with RPM $>10$ in either genotype are shown. The red dots indicate miRNAs showing increased abundance in rbv-1, and the blue dots indicate miRNAs with reduced abundance in rbv-1 (Student's $t$ test, ${ }^{\star} P<0.05$ ). b A scatter plot showing the AGO1 loading efficiency of miRNAs in rbv-1 vs. Col as determined by AGO1 IP small RNA-seq. AGO1 loading efficiency is represented by the ratio of miRNA abundance in AGO1 IP vs. input. All miRNAs with RPM value $>10$ in either genotype in the input samples (as in a) are shown here. The red dots indicate miRNAs with increased AGO1 association in $r b v-1$, and the blue dots indicate miRNAs with reduced AGO1 association in $r b v-1$ (Student's $t$ test, ${ }^{*} P<0.05$ ). c RNA gel blots analysis of three miRNAs before (input) and after AGO1 IP. U6 was used as an internal control for the input samples. For the IP samples, a portion was used for protein gel blot analysis to quantify AGO1 protein levels. The levels of miRNAs in the IP samples were normalized against AGO1 protein levels. No matter whether the assayed miRNAs were increased or reduced in abundance in input samples, they all showed reduced AGO1 association. Three independent repeats gave similar results. d Western blots to determine the nucleocytoplasmic partitioning of AGO1 in Col and rbv-1. T total extract, $\mathrm{C}$ cytoplasmic fraction, $\mathrm{N}$ nuclear fraction. Blots were analyzed using $\mathrm{AGO1}, \mathrm{GAPDH}$, and $\mathrm{H} 3$ antibodies, respectively. $\mathrm{H} 3$ was used as a nuclear marker in the quantification of AGO1 in the T and N samples. GAPDH was used as a cytoplasmic marker in the quantification of AGO1 in the T and C samples. Three independent repeats gave similar results. e Small-RNA gel blot analysis to determine the levels of miRNAs from total extract $(T)$ and from the cytoplasmic (C) and nuclear (N) fractions in Col and rbv-1. U6 and tRNAMet served as nuclear and cytoplasmic RNA markers, respectively. They also served as the loading controls for the nuclear and cytoplasmic fractions for the quantification of miRNA levels. Two independent repeats gave similar results. $\mathbf{f}$ Small RNA gel blot analysis of miRNAs in AGO1 IP from the cytoplasmic (C) and nuclear (N) fractions. NE, normal exposure; LE long exposure. Two independent repeats gave similar results. $\mathrm{g}$ Size exclusion chromatography with $p R B V$ :RBV-eYFP rbv-1 and rbv-1 samples followed by western blotting to detect AGO1 and northern blotting to detect miR159. The upper panel indicates the distribution of AGO1 while the lower panel represents the distribution of miR159 among the fractions. The numbers above the AGO1 blots indicate those of the fractions. Note that no AGO1 or miR159 was detected in fractions 1-7 (not shown). The positions of the molecular weight standards are shown above the AGO1 blots. Two independent repeats gave similar results. Source data are provided as a Source Data file.

loading efficiencies for miR159, miR166, miR156, and miR319 were similarly reduced in the nuclear and cytoplasmic fractions in the $r b v-1$ mutant, suggesting that this experiment at the steadystate level could not pinpoint the subcellular location of the AGO1 loading defect of the mutant. Intriguingly, for miR163 that showed increased abundance in both the nuclear and the cytoplasmic fractions in the mutant (Fig. 5e), the loading efficiencies were reduced in the nuclear but not the cytoplasmic fraction in $r b v-1$ (Fig. 5f), which is indicative of a nuclear loading defect.

To determine how RBV might promote the loading of miRNAs into AGO1, we first examined whether RBV interacts with AGO1. Co-IP was performed with $p R B V: R B V$-eYFP plants using antiGFP and anti-AGO1 antibodies, but no interaction between RBV and AGO1 was detected. We next sought to determine whether the bi-molecular feature of miRISCs is affected in the mutant. Protein extracts from $p R B V: R B V-e Y F P r b v-1$ and $r b v-1$ plants were subjected to gel filtration followed by western blotting to detect AGO1 and northern blotting to detect miR159. Among the 44 fractions, AGO1 was distributed in both high molecular weight (HMW) (fractions 9-11) and low molecular weight (LMW) (fractions 16-17) complexes in $p R B V: R B V$-eYFP rbv-1, consistent with previous findings ${ }^{28}$. Notably, the LMW complexes, which corresponded to monomeric AGO1 in size, also showed peak levels of miR159, suggesting that they represent miRISCs with one AGO1 protein and one miRNA. However, such miRISCs were greatly reduced in $r b v$-1, with AGO1 being only in HMW complexes instead (Fig. 5g). The distribution of miR159 also shifted towards HMW complexes in $r b v-1$ (Fig. $5 \mathrm{~g}$ ). Western blot analyses with anti-GFP antibody showed that RBV-eYFP was present in fractions 8-16 with estimated molecular weights much higher than that of an RBV-eYFP monomer, suggesting that RBV itself also resides in protein complexes (Supplementary Fig. 10). Therefore, RBV promotes the formation of miRISCs that contain only AGO1 and miRNAs. The HMW AGO1 complexes may represent intermediates in RISC formation.

AGO1 is known to associate with trans-acting small interfering RNAs (ta-siRNAs) in addition to miRNAs ${ }^{24,26}$. Contrary to miRNAs, which are loaded into AGO1 in the nucleus, ta-siRNAs are loaded into AGO1 in the cytoplasm ${ }^{25}$. We investigated whether RBV affects the ta-siRNA-AGO1 association. We quantified 21-nt siRNAs that mapped to 100-bp windows that overlapped with TAS1A, TAS1B, TAS1C, and TAS2 loci. The ta-
siRNAs were not significantly altered in $r b v$ - 1 input as compared to Col input in each 100-bp window (Supplementary Fig. 11a, c, e, g). We then analyzed their levels in AGO-IP vs. input and found that the loading of ta-siRNAs into AGO1 was largely unaffected. Only ta-siRNAs in window 3 of TAS $2 B$ and window 3 of TAS1C were affected (Supplementary Fig. $11 \mathrm{~b}, \mathrm{~d}, \mathrm{f}, \mathrm{h}$ ). The levels of miR173, the trigger of ta-siRNA biogenesis from TAS1 and TAS2 loci, were not significantly different between Col and the $r b v-1$ mutant (Supplementary Fig. 11i). The fact that tasiRNA loading was largely unaffected in the mutant is consistent with RBV being a nuclear protein.

$\mathrm{RBV}$ is required for the splicing of short introns in certain premRNAs. In order to determine whether $R B V$ affects the expression of protein-coding genes, we performed RNA-seq with 14day-old seedlings of Col and $r b v-1$ in triplicates. The three biological replicates for each genotype were highly reproducible (Supplementary Fig. 12).

Differentially expressed genes (DEGs) were identified between Col and mutant samples with FPKM $>1$, fold-change $>2$, and FDR $<0.05$ as the cutoff. In total, we identified 632 upregulated (hyper-DEGs) and 363 downregulated (hypo-DEGs) genes, respectively (Supplementary Data 5 and 6, Supplementary Fig. 13a). Gene Ontology (GO) analyses showed that both the hyper-DEGs and hypo-DEGs were enriched in genes with roles in responses to stimuli (Supplementary Fig. 13b, c). We also examined the global transcript levels of miRNA targets from the RNA-seq data. Although several examined miRNA targets were found to be derepressed in the mutant by RT-qPCR, a global trend of increased levels of miRNA target transcripts was not observed (Supplementary Fig. 13d, Supplementary Data 7). It is possible that the $r b v-1$ mutation leads to a defect in the transcriptional regulation of these genes as well.

It has been reported that MOS4-associated complex (MAC) components MAC3A, MAC3B, MAC7, and two WD40 repeat proteins (PRL1 and PRL2) affect both miRNA biogenesis and the splicing of protein-coding transcripts ${ }^{35-37}$. This prompted us to examine whether the $r b v-1$ mutant had splicing defects using the RNA-seq data (see Methods). In total, 474 Genes were found to have intron retention defects in the $r b v$-1 mutant compared to Col, with a total of 511 intron retention events (Supplementary Data 8). Two examples (At4g15790 and Atlg03280) are presented in Fig. 6a. PI (percent of intron reads) was increased in the $r b v-1$ 
a
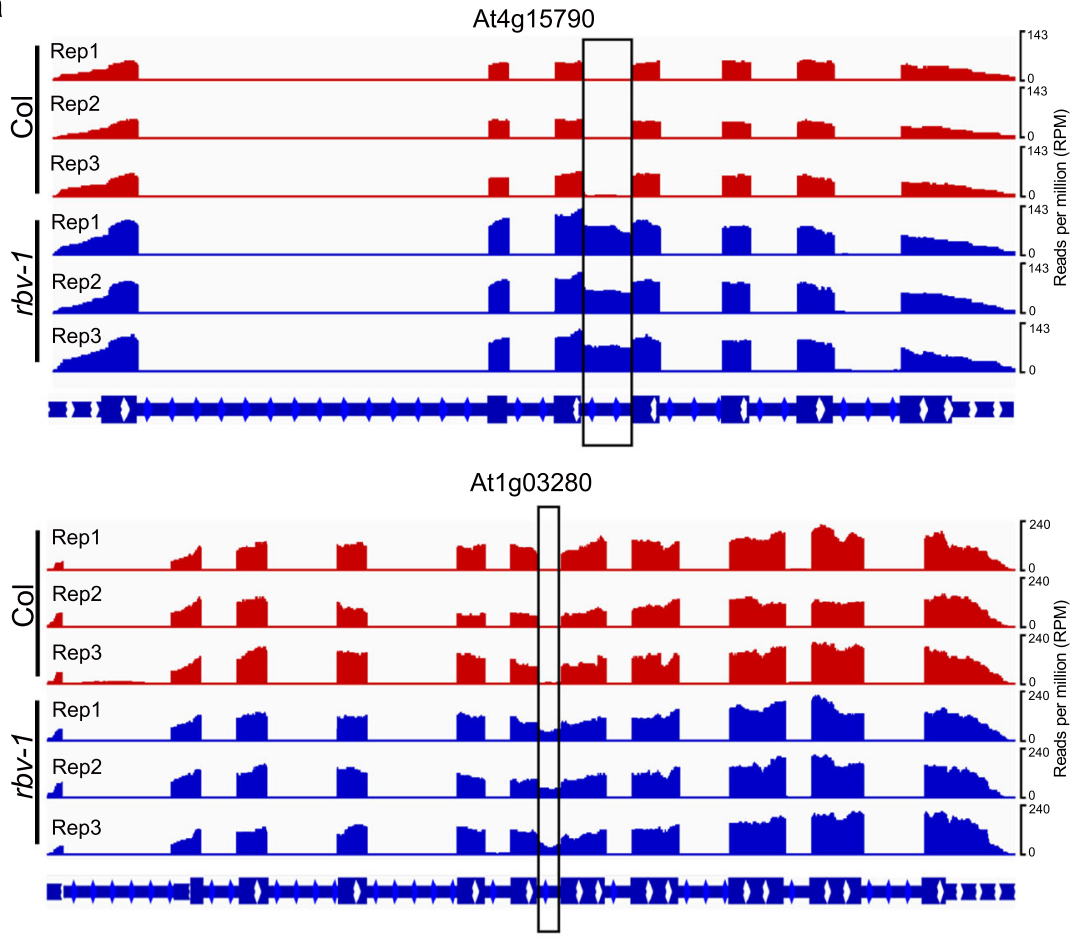

b

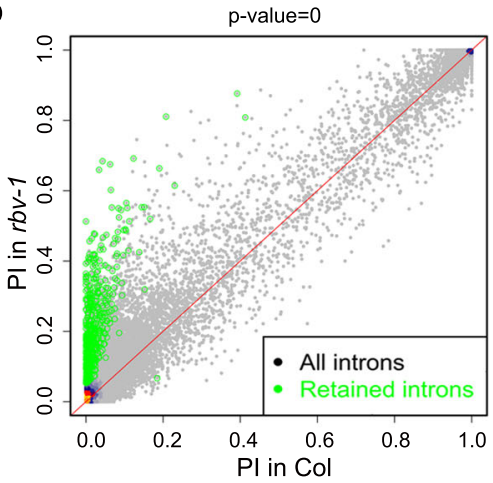

d

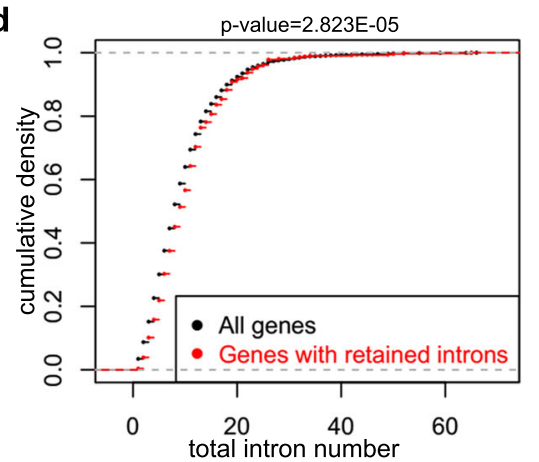

C

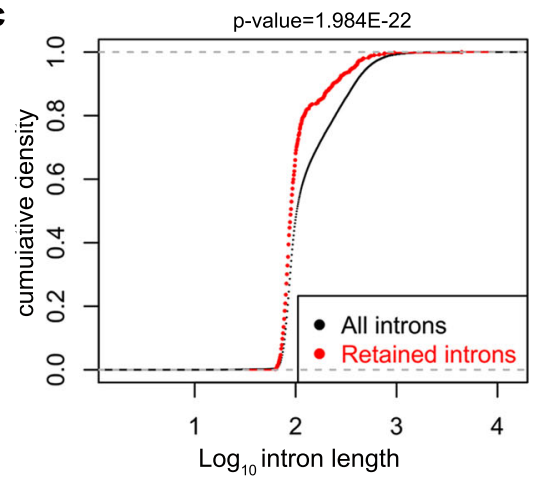

e

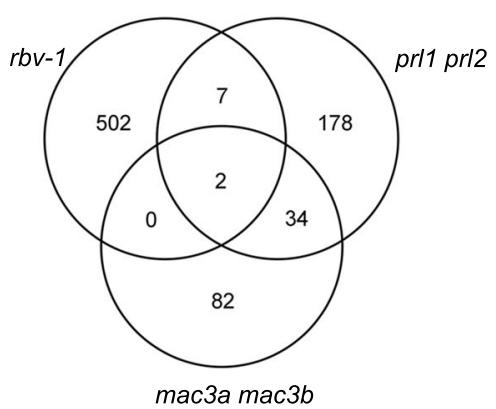

Fig. 6 RBV function is required in the splicing of short introns in certain pre-mRNAs. a Examples of two genes with intron retention defects in the $r b v-1$ mutant. RNA-seq reads are shown against the gene models below. In the gene models, rectangles and lines represent exons and introns, respectively. The black rectangles indicate retained introns in the rbv-1 mutant. b A scatter plot showing percent retained introns (PI) in wild type and the rbv-1 mutant. The green dots represent introns with statistically significant retention defects in the mutant (Wilcoxon test, $P=0$ ). c Cumulative density plots of intron length for all introns and for retained introns in the rbv-1 mutant (Wilcoxon test, $P=1.984 \mathrm{E}-22$ ). $\mathbf{d}$ Cumulative density plots of intron number in all genes and for genes with retained introns in the rbv-1 mutant. (Wilcoxon test, $P=2.823 \mathrm{E}-05$ ). e Venn diagrams showing the numbers of retained introns in $r b v-1$, prl1 prl2 and mac3a mac3b mutants, and the overlaps among the introns retained in these mutants. 


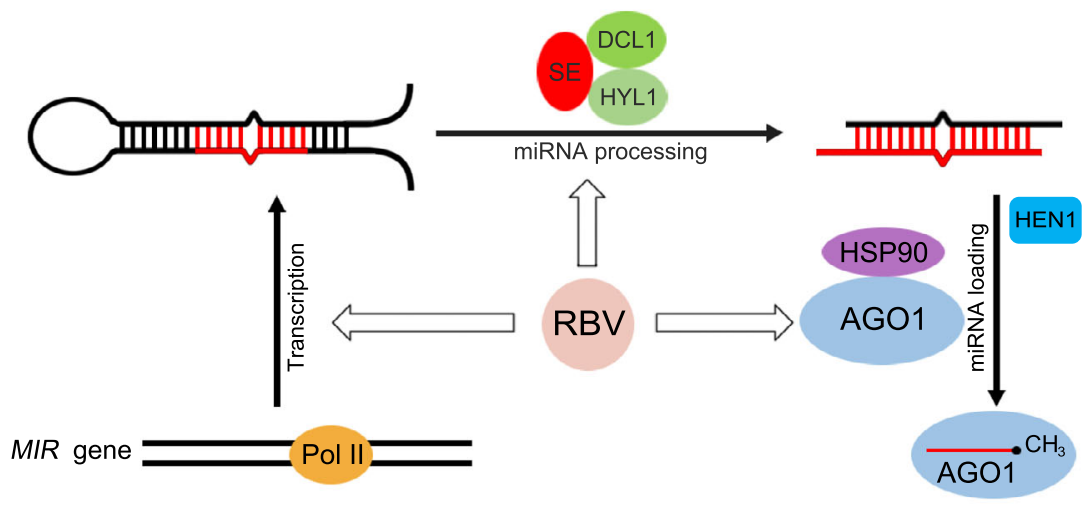

Fig. 7 A model for the role of $\boldsymbol{R B V}$ in miRNA biogenesis. A pathway of miRNA biogenesis entailing MIR gene transcription, pri-miRNA processing, miRNA methylation, and miRISC formation is shown. Key protein players in each step are depicted as ovals. RBV promotes miRNA biogenesis at the MIR gene transcription and AGO1 loading steps and may also enhance pri-miRNA processing.

mutant (Fig. 6b). No differential expression of the genes with intron retention was observed between the $r b v-1$ mutant and wild type (Supplementary Fig. 14a). Next, we examined whether the genes with intron retention or the retained introns in the $r b v-1$ mutant had any common features. Intriguingly, the retained introns in the $r b v-1$ mutant seemed to be shorter as compared to the overall length distribution of introns $(P=1.984083 \mathrm{e}-22)$ (Fig. 6c). Besides, genes affected in $r b v-1$ tended to have more introns than all genes $(P=2.823 \mathrm{e}-05)$ (Fig. 6d). Intron retention defects are also found in prl1 prl2 and mac3a mac3b mutants ${ }^{35}$. We compared the retained introns in $r b v-1$, prl1 prl2 and mac $3 a$ $m a c 3 b$ and found little overlap between the introns affected in $r b v$-1 with those affected in either prl1 prl2 or mac3a mac3b (Fig. 6e), suggesting that $R B V$ affects different introns from the MAC components.

Many $M I R$ genes have introns ${ }^{38-40}$. The RNA-seq did not detect intron retention events in pri-miRNAs, but the low abundance of pri-miRNAs could have precluded the detection of intron retention events. We performed RT-PCR to test the splicing of introns from three miRNA precursors (pri-miR163, pri-miR156, and pri-miR168) with intron-flanking primers. Genomic DNA was amplified with the same primers to indicate the size of the intron-containing fragments. No defects in primiRNA splicing were observed in the $r b v$ - 1 mutant (Supplementary Fig. 14b).

\section{Discussion}

RBV, a WD40 protein, is an evolutionarily conserved protein in plants. However, it has not been studied from any species. In this study, we show that a recessive mutation in $R B V$ reduces the levels of many endogenous miRNAs, indicating that mutation of RBV leads to a defect in miRNA biogenesis. Consistent with the known roles of plant miRNAs in various developmental processes, the $r b v-1$ mutant exhibits strong developmental defects. How does $R B V$ promote miRNA biogenesis? Our findings suggest a role of $R B V$ in promoting Pol II transcription of $M I R$ genes, which is supported by the reduced levels of pri-miRNAs, compromised MIR167a promoter activity, and decreased Pol II occupancy at MIR genes in the $r b v-1$ mutant. In Arabidopsis, a series of proteins have been found to promote $M I R$ transcription and/or pri-miRNA processing and can be grouped into two main classes $^{1}$. One group contains CBP80 and CBP20 $41, \mathrm{STA}^{42}$, SICKLE ${ }^{39}$, TOUGH $^{43}, \mathrm{PINP}^{44}$, THO1 and $\mathrm{THO}^{45,46}$, and MOS2 ${ }^{47}$. A common feature is that mutants in these genes show reduced levels of miRNAs and increased abundance of pri-
miRNAs. The second group of proteins acts in a different manner. In loss-of-function mutants in the genes in this group, the abundance of both pri-miRNAs and mature miRNAs is reduced. Proteins in this group include $\mathrm{DAWDLE}^{48}, \mathrm{CDC}^{7}, \mathrm{NOT}^{6}$, Elongator ${ }^{8}, \mathrm{PRL}_{1}^{49}, \mathrm{MAC7}^{35}, \mathrm{PP} 4^{37}$, and $\mathrm{THP}^{9}$. Besides, mutants in PRL1, CDC5, MAC7, PP4, and THP1 show a reduced number of HYL1 D-bodies $7,9,35,37,49$. A number of proteins in this group interact with DCL1, HYL1, or SE and thus are thought to bridge MIR gene transcription and pri-miRNA processing ${ }^{6-9,37,48}$. Besides the two main classes, the third group, which includes CHR2, a partner of SE, promotes the transcription of MIR genes but represses miRNA accumulation by inhibiting pri-miRNA processing ${ }^{10}$. Our studies show that RBV belongs to the second group of proteins that promotes $M I R$ gene transcription and possibly pri-miRNA processing.

$\mathrm{RBV}$ also differs from the second group of proteins in that it has a clear role in miRISC formation. In the $r b v$ - 1 mutant, both AGO1 and miR159 shift into complexes with higher molecular weights. RBV itself is also found in HMW complexes. We suspect that the HMW complexes containing AGO1 and miR159 represent intermediates in miRISC formation and that RBV helps the dissociation of AGO1-miRNA from other proteins to form active miRISCs. Heat Shock Protein 90 (HSP90) is required for sRNA loading into AGO1 in tobacco lysates ${ }^{50}$ and for RISC formation in Drosophila and humans ${ }^{51}$. HSP90 proteins are involved in RNA silencing in animals ${ }^{52}$ and plants ${ }^{53,54}$. Consistently, HSP90.4 was found in GFP-AGO1 IP spectrometry experiments ${ }^{25}$. Another protein that has recently been shown to play a role in miRISC formation is CARP9, which interacts with both HYL1 and AGO1 in the nucleus ${ }^{55}$. The relationship between RBV, HSP90 and CARP9 is worth investigating in the future. In summary, RBV, as a nuclear WD40 protein, is involved in multiple steps in miRNA biogenesis, including $M I R$ transcription, pri-miRNA processing and AGO1 loading (Fig. 7).

Proteins such as MAC7, PP4, MAC3A/3B, and PRL1 act not only in miRNA biogenesis but also in pre-mRNA splicing in Arabidopsis ${ }^{35,37}$. We found that RBV also plays a role in splicing. Similar to mutants in $M A C 7, P P 4, M A C 3 A / 3 B$, and $P R L 1$, no significant correlations between intron retention and changes in gene expression were found in the $r b v-1$ mutant. Introns retained in the $r b v$ - 1 mutant had minimal overlap with those in the other mutants, suggesting the presence of different categories of introns, whose splicing requires distinct factors. $R B V$ acts on genes with more introns and introns that are shorter in length. Furthermore, intron retention was not found for introncontaining pri-miRNAs in $r b v-1$, suggesting that mutation of 
$R B V$ leads to a defect in miRNA biogenesis and pre-mRNA splicing independently. It is possible that $R B V$ function is required for yet other aspects of nuclear RNA metabolism.

\section{Methods}

Plant materials and growth conditions. The transgenic Arabidopsis thaliana (Col ecotype) line $p S U C 2: a m i R-S U L$ expressing amiR-SUL under the sucrose-proton symporter 2 (SUC2) promoter was a kind gift from Dr. Detlef Weige ${ }^{29}$. Transgenic lines harboring pSE:SE-mRuby3, pMIR167a:GUS, and pHYL1:HYL1-YFP 5,9,22,49 were used in this study. All seeds were sown on 1/2 Murashige and Skoog Basal Medium (Sigma-Aldrich, M5519) plus 1\% Sucrose and 0.8\% Agar. All plants were grown at $22^{\circ} \mathrm{C}$ under $16 \mathrm{~h}$ light and $8 \mathrm{~h}$ dark cycles.

Mutagenesis and mapping. EMS mutagenesis was performed as described ${ }^{9}$. A mutant, amiR-SUL $r b v$-1, with reduced leaf bleaching, was isolated and then backcrossed with the parental line pSUC2:amiR-SUL. In the F2 generation, 100 plants with the amiR-SUL $r b v-1$ phenotype were identified. Genomic DNA was extracted by the CTAB method ${ }^{56}$ from individual plants and an equal amount of DNA was pooled for genomic DNA library construction. The library was pairedend (PE151bp) sequenced on the Illumina platform HiSeq4000 at 50x coverage at BGI-Shenzhen, China. Focusing only on G-to-A mutations in coding regions, a mutation in At5g64730 was identified in amiR-SUL $r b v$-1. A derived cleaved amplified polymorphic sequences (dCAPS) marker was designed to genotype this mutation (Supplementary Table 2). The PCR products from wild type can be digested by NcoI, whereas those from amiR-SUL rbv-1 could not. Genotyping the $\sim 100$ individuals showed that this mutation was linked to the plant phenotype.

DNA constructs and complementation. The genomic region of $R B V$ (At5g64730) including $\sim 1 \mathrm{~kb}$ promoter was amplified with the primers proRBV-F and RBV-R (Supplementary Table 2) and cloned into the pTSK108 vector. The clone was sequenced to ensure the absence of mutations and was recombined with the pGWB640 gateway vector ${ }^{57}$ to generate the $p R B V: R B V-e Y F P$ construct via LR reaction. The $p R B V: R B V-e Y F P$ plasmid was transformed into the $r b v-1$ mutant in both amiR-SUL and Col backgrounds through the A. tumefaciens-mediated floral dip method as described ${ }^{58}$. The T1 transgenic plants were selected by BASTA resistance.

Fluorescence microscopy. For the visualization of RBV-eYFP and HYL1-YFP in transgenic plants, roots of 10-day-old seedlings were observed under a Zeiss LSM 5 Pascal inverted confocal microscope (excitation, $488 / 512 \mathrm{~nm} / \mathrm{nm}$; emission, 520/ $50 \mathrm{~nm} / \mathrm{nm}$ ). For SE-mRuby3 and SE-eCFP, roots of 10-day-old $p S E: S E-m R u b y 3$ seedlings were observed under a LeicaSP8 confocal microscope (excitation, $514 \mathrm{~nm}$; emission, $540-600 \mathrm{~nm}$ ).

Small RNA sequencing and data analysis. Total RNAs were extracted from 14day-old seedlings using TRI reagent (TR118, Molecular Research Centre). Small RNAs in the size range of $15-40 \mathrm{nt}$ were isolated from $30 \mu \mathrm{g}$ total RNAs by resolving total RNAs in denaturing polyacrylamide gels, excising gel pieces containing 15-40 nt RNAs and elution of the small RNAs according to Liu et al ${ }^{59}$. Small RNA libraries were prepared with NEBNext Multiplex Small RNA Library Prep Set for Illumina (New England Biolabs, E7300), and then sequenced using an Illumina HiSeq2500 platform at BerryGenomics, China. Data analysis was performed with the pRNASeqTools pipeline (https://github.com/grubbybio/ pRNASeqTools). The raw reads (SE50) were trimmed using Perl scripts to remove adapters (adapter: AGATCGGAAGAGC). The clean reads were mapped to the $A$. thaliana genome using the Bowtie program ${ }^{60}$. For miRNA analysis, the small RNA reads were mapped to miRBase v21. The sequences of miRNA and miRNA* were obtained from the PRIMEN database: http://www.pmiren.com/ftpdownload/ Arabidopsis_thaliana_Ath/Arabidopsis_thalianamature.fa. For tasiRNA analysis, the reads that were mapped to 100 -bp windows overlapping with $T A S 1 A / B / C$ and TAS2 loci were counted. The small RNA reads were normalized by calculating the RPM value (reads per million trimmed reads) ${ }^{61}$. The comparison between genotypes was conducted by the R package "DESeq2" with a 1.5 -fold change and $P<0.01$ as the cutoff 6 . For AGO1 IP sRNA-seq analysis, reads were normalized by total mapped reads. The comparison between genotypes was conducted with the two-factor model by DESeq2.

Small RNA gel blotting. RNA gel analysis of small RNAs was performed as described $^{63}$. Ten micrograms of total RNAs were separated on $15 \%$ polyacrylamide/ $8 \mathrm{M}$ urea gels. After gel electrophoresis, the RNAs were transferred to a Hybond-NX nylon membrane (GE healthcare). Antisense complementary oligonucleotides (Supplementary Table 2) were synthesized with both $5^{\prime}$ and $3^{\prime}$ end-labeled biotin. A probe complementary to U6 ( $5^{\prime}$ CATCCTTGCGCAGGGGCCA $\left.3^{\prime}\right)$ was used to detect $\mathrm{U} 6$ as an internal control. Hybridization was performed for $16 \mathrm{~h}$ at $55^{\circ} \mathrm{C}$ followed by washes. Signals were detected using the chemiluminescent nucleic acid detection module (Thermo Fisher, 89880).
Reverse transcription-quantitative PCR (RT-qPCR). RT-qPCR was performed to quantify mRNA and pri-miRNAs levels. One microgram total RNA was reverse transcribed with oligo (dT) using the PrimeScript ${ }^{*} 1$ st Strand cDNA Synthesis Kit (TAKARA, 6110A) according to the manufacturer's instructions. RT-qPCR was performed in the 96-well StepOnePlus real-time system (ABI) using the SYBR premix ExTaq II kit (TAKARA, RR820A). The following scheme was used: $95^{\circ} \mathrm{C}$ for $30 \mathrm{~s}$ and $40 \mathrm{cycles}$ of $95^{\circ} \mathrm{C}$ for $5 \mathrm{~s}$ and $60^{\circ} \mathrm{C}$ for $30 \mathrm{~s}$. The levels of transcripts were normalized to the level of the internal standard UBQ5 and the $2^{-} \triangle \triangle \mathrm{CT}$ values of control samples were set to 1 . The primers for pri-miRNAs are listed in Supplementary Table 2. Three independent biological replicates were carried out for each genotype. The student's $t$-test was used for the evaluation of statistical significance.

Histochemical GUS staining. Fourteen-day-old seedlings of pMIR167a:GUS and pMIR167a:GUS rbv-1 (homozygous for both the transgene and the $r b v-1$ mutation) were subjected to histochemical GUS staining according to the standard protocol ${ }^{64}$ Tissues were vacuum infiltrated in the staining solution ( $1 \mathrm{mM}$ EDTA, $5 \mathrm{mM}$ potassium ferricyanide, $5 \mathrm{mM}$ potassium ferrocyanide, $100 \mathrm{mM}$ sodium phosphate, $1 \%$ Triton X-100, $1 \mathrm{mg} \mathrm{ml}^{-1} \mathrm{X}$-Gluc) for $10 \mathrm{~min}$ and then incubated at $37^{\circ} \mathrm{C}$ for $2 \mathrm{~h}$ in the dark. Tissue clearing was performed with $70 \%$ ethanol for $2 \mathrm{~h}$ before imaging.

Chromatin immunoprecipitation (ChIP) assay. RNA Polymerase II ChIP was performed as described ${ }^{5}$ with 14-day-old $\mathrm{Col}$ and $r b v$-1 seedlings using an antibody against RPB1 (Abcam, ab817, dilution 1:200). Seedlings were crosslinked in buffer $(0.4 \mathrm{M}$ Sucrose, $10 \mathrm{mM}$ Tris-HCl Ph8.0, $1 \mathrm{mM}$ EDTA, $1 \mathrm{mM}$ PMSF, $1 \%$ proteinase inhibitor cocktail, and $1 \%$ formaldehyde) at room temperature under the vacuum and glycine was added to a final concentration of $100 \mathrm{mM}$ to stop the crosslinking. Then the plant material was ground to a fine powder, which was then resuspended in cold nuclei-isolation buffer $(0.25 \mathrm{M}$ sucrose, $15 \mathrm{mM}$ PIPES pH $6.8,5 \mathrm{mM} \mathrm{MgCl} 2,60 \mathrm{mM} \mathrm{KCl}, 15 \mathrm{mM} \mathrm{NaCl}, 1 \mathrm{mM}$ $\mathrm{CaCl}_{2}, 0.9 \%$ Triton X-100, $1 \mathrm{mM}$ PMSF and $1 \%$ proteinase inhibitor cocktail). The suspension was filtered through two layers of Miracloth and centrifuged at $12,000 \mathrm{~g}$ for $20 \mathrm{~min}$ at $4{ }^{\circ} \mathrm{C}$. The pelleted nuclei were resuspended in nuclei lysis buffer (50 mM HEPES pH7.5, $150 \mathrm{mM} \mathrm{NaCl}, 1 \mathrm{mM}$ EDTA, 1\% sodium dodecyl sulfate (SDS), $0.1 \%$ sodium deoxycholate, $1 \%$ Triton X-100, and $1 \%$ proteinase inhibitor cocktail) followed by sonication (Covaris S200). Then the sonicated suspension was centrifuged at $12,000 \mathrm{~g}$ at $4{ }^{\circ} \mathrm{C}$ to pellet debris. The supernatant was collected and diluted with ChIP dilution buffer (50 mM HEPES pH7.5, $150 \mathrm{mM} \mathrm{NaCl}, 1 \mathrm{mM}$ EDTA, $0.1 \%$ sodium deoxycholate, $1 \%$ Triton X-100, and $1 \%$ proteinase inhibitor cocktail). The diluted chromatin sample was precleared with protein A agarose beads followed by incubation with antibodies with gentle rotation overnight at $4^{\circ} \mathrm{C}$. Then protein A agarose beads were added followed by incubation for $2 \mathrm{~h}$ at $4{ }^{\circ} \mathrm{C}$ with gentle rotation. The beads were washed with low-salt wash buffer $(150 \mathrm{mM} \mathrm{NaCl}, 20 \mathrm{mM}$ Tris- $\mathrm{HCl} \mathrm{pH} 8.0$, $0.2 \%$ SDS, $0.5 \%$ Triton X-100, and $2 \mathrm{mM}$ EDTA), high-salt wash buffer $(500 \mathrm{mM} \mathrm{NaCl}, 20 \mathrm{mM}$ Tris-HCl pH8.0, 0.2\% SDS, $0.5 \%$ Triton X-100 and $2 \mathrm{mM}$ EDTA $)$, and $\mathrm{LiCl}$ wash buffer $(0.25 \mathrm{M} \mathrm{LiCl}, 1 \%$ sodium deoxycholate, $10 \mathrm{mM}$ Tris-HCl pH8.0, 1\% CA630 and 1 mM EDTA) and twice with TE buffer (1 mM EDTA, $10 \mathrm{mM}$ Tris- $\mathrm{HCl}$ pH8.0). The chromatin was eluted with elution buffer $(1 \mathrm{mM} \mathrm{NaHCO}$ and $1 \%$ SDS $)$ and $20 \mu \mathrm{l} 5 \mathrm{M} \mathrm{NaCl}$ was added followed by incubation at $65^{\circ} \mathrm{C}$ for at least $6 \mathrm{~h}$ to reverse crosslinking. Removal of protein and RNA was achieved by adding $1.5 \mu \mathrm{l} 18.9 \mathrm{mg} \mathrm{ml}^{-1}$ proteinase $\mathrm{K}$ and $1 \mu \mathrm{l}$ of $1 \mathrm{M}$ RNase A per $500 \mu \mathrm{l}$. DNA was extracted by the phenol/chloroform extraction method. RT-qPCR was performed with co-immunoprecipitated DNA, using primers listed in Supplementary Table 2. Relative enrichment was calculated by normalizing the amount of ChIP-ed DNA to the corresponding amount in the input.

Western blot analysis. Western blots were performed as described ${ }^{63}$. Proteins from 14-day-old seedlings were extracted, resolved in $12 \%(\mathrm{v} / \mathrm{v})$ SDS polyacrylamide gels, and transferred to Hybond C-Extra membranes (Amersham Biosciences). The membranes were blocked with $5 \%(\mathrm{w} / \mathrm{v})$ non-fat milk in Tris buffered saline tween (TBST) buffer and then probed with specific antibodies. Antibodies used included anti-GAPDH (Santa Cruz Biotechnology, sc-365062, dilution, 1:1000), anti-SUL (dilution, 1:1000) ${ }^{35}$, anti-HYL1 (Agrisera, AS06136, dilution, 1:2000), anti-AGO1 (Agrisera, AS09527, dilution, 1:2000), anti-SE (Agrisera, AS09532A, dilution, 1:1000), anti-H3 (Agrisera, AS10710, dilution 1:3000) and anti-GFP (Abcam, ab290, dilution, 1:3000). After three washes, the membranes were probed with horseradish peroxidase-conjugated, goat-anti-rabbit IgG (Bio-Rad, cat.172-1019) (dilution, 1:2000) or goat anti-mouse IgG (Bio-Rad, cat.170-6516, dilution, 1:2000). The protein signals were detected with the Amersham ${ }^{\mathrm{TM}} \mathrm{ECL}^{\mathrm{TM}}$ Prime Western Blotting Detection Reagent (GE healthcare, RPN2232) and visualized with the Chemiluminescence imaging system (Clinx Science Instruments Co. Ltd., China).

RNA-Seq data analysis. Total RNAs extracted from 14-day-old seedlings were sent for library construction at Novogene, China and libraries were sequenced on an Illumina Hiseq 4000 platform to generate paired-end reads of $150 \mathrm{bp}$ in 
length. The data analysis of RNA-seq libraries was carried out as described ${ }^{65}$ The clean reads were collapsed into nonredundant ones and mapped to the Arabidopsis genome (ARAPORT11) using STAR, allowing a maximum of eight mismatches per paired-end read ${ }^{66}$. DEGs were identified between $\mathrm{Col}$ and $r b v$ - 1 using cuffdiff ${ }^{67}$ with FPKM $>1$, fold-change $>2$ and FDR $<0.05$ as filters. Expression levels of all genes and DEGs were plotted using the value of $\log 2$ $(\mathrm{FPKM}+1) . \mathrm{GO}$ enrichment analysis was performed with agriGO ${ }^{68}$ using a webtool (http://bioinfo.cau.edu.cn/agriGO/). Only the top 20 terms were presented in this paper. The analysis of splicing defects was carried out using Araport 11 intron annotation and a previously developed pipeline known as SQUID (https://github.com/sfli001/SQUID). In brief, the level of retained introns was calculated using two methods: PI_Junction (intron-exon junction reads/[intron-exon junction reads + exon-exon reads]) and PI_density (normalized intronic reads/normalized exonic reads). The differentially spliced introns were defined using a stringent cutoff: combined_FDR $<0.1$, Diff_PI_Junction $>0.05$, Diff_PI_Density $>0.05$. PI_Junction was used to represent the levels of retained introns.

AG01 IP assay. One gram of 14-day-old seedlings was ground in liquid nitrogen, and IP buffer (50 mM Tris pH 7.5, $150 \mathrm{mM} \mathrm{NaCl}, 10 \%$ Glycerol, 0.1\% NP-40, $1 \mathrm{mM}$ PMSF) and EDTA-free protease inhibitor mixture (Roche) were added to the powder, which was followed by $20 \mathrm{~min}$ incubation with gentle shaking at $4{ }^{\circ} \mathrm{C}$. The supernatant was incubated with $100 \mu \mathrm{L}$ of dynabeads (Invitrogen, 10002D) for $2 \mathrm{~h}$ at $4{ }^{\circ} \mathrm{C}$. After centrifugation, the supernatant was used for IP. $50 \mu \mathrm{L}$ was saved as input, and the rest was incubated with anti-AGO1 antibody (Agrisera, AS09527, dilution, 1:2000) for $2 \mathrm{~h}$ at $4{ }^{\circ} \mathrm{C}$. The beads were washed with wash buffer (IP buffer with $0.5 \% \mathrm{NP}-40)$, and $1 / 10(\mathrm{v} / \mathrm{v})$ was added to $2 \times$ SDS-loading buffer for western blot analysis and 9/10 (v/v) was used for RNA isolation. Small RNA libraries were prepared using the NEBNext Multiplex Small RNA Library Prep Set for Illumina (New England Biolabs, E7300), and sequenced on an Illumina HiSeq2500 platform at BerryGenomics, China. The proteins were separated by SDS-PAGE and protein gel blot analysis was performed using anti-AGO1 antibody (Agrisera, AS09527, dilution, 1:2000).

Nuclear-cytoplasmic fractionation. Twelve-day-old seedlings were collected and cross-linked in $0.5 \%$ formaldehyde/ $1 \times$ phosphate-buffered saline (PBS) buffer under vacuum for $10 \mathrm{~min}$ twice on ice. Then glycine was added to a final concentration of $100 \mathrm{mM}$ to stop the crosslinking followed by incubation for $5 \mathrm{~min}$ under vacuum on ice. The plant material was washed in $1 \times$ PBS buffer and frozen in liquid nitrogen immediately for nuclear-cytoplasmic fractionation. ${ }^{9}$ The frozen seedlings were ground to a fine powder in liquid nitrogen and resuspended in $2 \mathrm{ml} \mathrm{g}^{-1}$ lysis buffer (20 mM Tris-HCl, pH7.5, $20 \mathrm{mM}$ EDTA, $2.5 \mathrm{mM} \mathrm{MgCl}, 25 \%$ glycerol, $250 \mathrm{mM}$ sucrose, $5 \mathrm{mM}$ DTT, and $1 \times$ protease inhibitor cocktail (Roche)). The suspension was filtered through two layers of Miracloth. The flow-through was centrifuged at $1500 \mathrm{~g}$ for $10 \mathrm{~min}$ at $4^{\circ} \mathrm{C}$. The supernatant was centrifuged at $10,000 \mathrm{~g}$ for $10 \mathrm{~min}$ at $4{ }^{\circ} \mathrm{C}$, and the supernatant was collected as the cytoplasmic fraction, which was used for RNA isolation using the Trizol method. The pellet from the $1500 \mathrm{~g}$ spin was washed 10 times with $10 \mathrm{ml}$ nuclear resuspension buffer 1 (NRB1) (20 mM Tris- $\mathrm{HCl}, \mathrm{pH} 7.5,2.5 \mathrm{mM} \mathrm{MgCl}_{2}$, and $0.2 \%$ Triton X-100). The washed pellet was resuspended with $500 \mu \mathrm{l} \mathrm{NRB} 2\left(20 \mathrm{mM}\right.$ Tris-HCl, pH7.5, $10 \mathrm{mM} \mathrm{MgCl}_{2}$, $250 \mathrm{mM}$ sucrose, $0.5 \%$ Triton X-100, $5 \mathrm{mM} \beta$-mercaptoethanol, and $1 \times$ protease inhibitor cocktail). The sample was centrifuged at $16,000 \mathrm{~g}$ for $45 \mathrm{~min}$ at $4{ }^{\circ} \mathrm{C}$. The final nuclear pellet was used for RNA isolation using the Trizol method. The cytoplasmic and nuclear fractions underwent the same AGO1 IP procedure as described above.

Gel filtration assay. One gram of 14-day-old T3 $p R B V$ : $R B V$-eYFP $r b v$-1 seedlings and wild type was collected and ground in liquid nitrogen. Then $1.5 \mathrm{ml}$ phosphate buffer supplemented with $1 \mathrm{mM}$ PMSF, 1\% EDTA-free protease inhibitor mixture, RNase Inhibitor (TAKARA) and $0.4 \%$ CA630 were added to the powder. The homogenized crude extracts were kept on ice for $20 \mathrm{~min}$. After two rounds of centrifugation for $20 \mathrm{~min}(12,000 \mathrm{~g})$ each at $4{ }^{\circ} \mathrm{C}, 1.5 \mathrm{ml}$ of the cleared crude extract was immediately used for sample injection. Gel-filtration chromatography was carried out as described ${ }^{28}$ using a Superdex 200 Increase 10/300GL column. In total, 44 fractions (each $800 \mu \mathrm{L}$ ) were collected. A $300 \mu \mathrm{L}$ of each fraction was used for protein extraction for detecting AGO1 and $500 \mu \mathrm{L}$ for total RNA purification for sRNA detection. Markers of known sizes (Aprotinin, $6.5 \mathrm{kDa}$; Ribonuclease A, $13.7 \mathrm{kDa}$; Carbonic Anhydrase, $29 \mathrm{kDa}$; Conalbumin, $75 \mathrm{kDa}$; Aldolase, $158 \mathrm{kDa}$; Ferritin, $440 \mathrm{kDa}$; Thyroglobulin, $669 \mathrm{kDa}$; blue dextran, $2000 \mathrm{kDa}$ ) were used for column calibration.

RBV conservation analysis. hmmsearch (HMMER 3.3) was employed to search for RBV homologs in a plant genome database (Supplementary Data 2) with a seed file created by the cDNA sequence of $R B V$. Close homologs of $R B V$ were retained by a primary Neighbor-Joining tree based on MUSCLE (v3.8.1551)-aligned sequences and manual selection. The coding sequences of these genes were aligned by MUSCLE and manually corrected.
The final maximum likelihood tree was constructed using the general timereversible model and 1000 bootstrap replicates based on nucleotide sequences. The output tree was visualized by iTOL ${ }^{69}$.

Reporting summary. Further information on research design is available in the Nature Research Reporting Summary linked to this article.

\section{Data availability}

All raw and processed data were deposited at NCBI GEO (http://www.ncbi.nlm.nih.gov/ geo/) with the accession number GSE152911. Source data are provided with this paper

Received: 17 May 2021; Accepted: 8 February 2022;

Published online: 08 March 2022

\section{References}

1. Yu, Y., Jia, T. \& Chen, X. The 'how' and 'where' of plant microRNAs. $N$. Phytol. 216, 1002-1017 (2017).

2. Song, X., Li, Y., Cao, X. \& Qi, Y. MicroRNAs and their regulatory roles in plant-environment interactions. Annu. Rev. Plant Biol. 70, 489-525 (2019).

3. Xie, Z. et al. Expression of Arabidopsis MIRNA genes. Plant Physiol. 138, 2145-2154 (2005).

4. Zheng, B. et al. Intergenic transcription by RNA polymerase II coordinates Pol IV and Pol V in siRNA-directed transcriptional gene silencing in Arabidopsis. Genes Dev. 23, 2850-2860 (2009).

5. Kim, Y. J. et al. The role of mediator in small and long noncoding RNA production in Arabidopsis thaliana. EMBO J. 30, 814-822 (2011).

6. Wang, L. et al. NOT2 proteins promote polymerase II-dependent transcription and interact with multiple MicroRNA biogenesis factors in Arabidopsis. Plant Cell 25, 715-727 (2013).

7. Zhang, S., Xie, M., Ren, G. \& Yu, B. CDC5, a DNA binding protein, positively regulates posttranscriptional processing and/or transcription of primary microRNA transcripts. Proc. Natl Acad. Sci. USA 110, 17588-17593 (2013).

8. Fang, X., Cui, Y., Li, Y. \& Qi, Y. Transcription and processing of primary microRNAs are coupled by elongator complex in Arabidopsis. Nat. Plants 1, 15075 (2015).

9. Zhang, B. et al. Linking key steps of microRNA biogenesis by TREX-2 and the nuclear pore complex in Arabidopsis. Nat. Plants 6, 957-969 (2020).

10. Wang, Z. et al. SWI2/SNF2 ATPase CHR2 remodels pri-miRNAs via Serrate to impede miRNA production. Nature 557, 516-521 (2018).

11. Reinhart, B. J., Weinstein, E. G., Rhoades, M. W., Bartel, B. \& Bartel, D. P. MicroRNAs in plants. Genes Dev. 16, 1616-1626 (2002).

12. Park, W., Li, J., Song, R., Messing, J. \& Chen, X. CARPEL FACTORY, a Dicer homolog, and HEN1, a novel protein, act in microRNA metabolism in Arabidopsis thaliana. Curr. Biol. 12, 1484-1495 (2002).

13. Fukudome, A. \& Fukuhara, T. Plant dicer-like proteins: double-stranded RNA-cleaving enzymes for small RNA biogenesis. J. Plant Res. 130, 33-44 (2017).

14. Morlando, M. et al. Primary microRNA transcripts are processed cotranscriptionally. Nat. Struct. Mol. Biol. 15, 902-909 (2008).

15. Pawlicki, J. M. \& Steitz, J. A. Primary microRNA transcript retention at sites of transcription leads to enhanced microRNA production. J. Cell Biol. 182, 61-76 (2008).

16. Vazquez, F., Gasciolli, V., Crete, P. \& Vaucheret, H. The nuclear dsRNA binding protein HYL1 is required for MicroRNA accumulation and plant development, but not posttranscriptional transgene silencing. Curr. Biol. 14, 346-351 (2004).

17. Han, M. H., Goud, S., Song, L. \& Fedoroff, N. The Arabidopsis doublestranded RNA-binding protein HYL1 plays a role in microRNA-mediated gene regulation. Proc. Natl Acad. Sci. USA 101, 1093-1098 (2004).

18. Yang, L., Liu, Z. Q., Lu, F., Dong, A. W. \& Huang, H. SERRATE is a novel nuclear regulator in primary microRNA processing in Arabidopsis. Plant J. 47, 841-850 (2006)

19. Lobbes, D., Rallapalli, G., Schmidt, D. D., Martin, C. \& Clarke, J. SERRATE: a new player on the plant microRNA scene. EMBO Rep. 7, 1052-1058 (2006).

20. Song, L., Han, M. H., Lesicka, J. \& Fedoroff, N. Arabidopsis primary microRNA processing proteins HYL1 and DCL1 define a nuclear body distinct from the Cajal body. Proc. Natl Acad. Sci. USA 104, 5437-5442 (2007).

21. Iwata, Y., Takahashi, M., Fedoroff, N. V. \& Hamdan, S. M. Dissecting the interactions of SERRATE with RNA and DICER-LIKE 1 in Arabidopsis microRNA precursor processing. Nucleic Acids Res. 41, 9129-9140 (2013).

22. Fang, Y. \& Spector, D. L. Identification of nuclear dicing bodies containing proteins for microRNA biogenesis in living Arabidopsis plants. Curr. Biol. 17, 818-823 (2007) 
23. $\mathrm{Yu}, \mathrm{B}$. et al. Methylation as a crucial step in plant microRNA biogenesis. Science 307, 932-935 (2005)

24. Baumberger, N. \& Baulcombe, D. C. Arabidopsis ARGONAUTE1 is an RNA Slicer that selectively recruits rnicroRNAs and short interfering RNAs. Proc. Natl Acad. Sci. USA 102, 11928-11933 (2005).

25. Bologna, N. G. et al. Nucleo-cytosolic shuttling of ARGONAUTE1 prompts a revised model of the plant microRNA pathway. Mol. Cell 69, 709-719 e705 (2018).

26. Qi, Y., Denli, A. M. \& Hannon, G. J. Biochemical specialization within Arabidopsis RNA silencing pathways. Mol. Cell 19, 421-428 (2005).

27. Wang, H. et al. Deep sequencing of small RNAs specifically associated with Arabidopsis AGO1 and AGO4 uncovers new AGO functions. Plant J. 67, 292-304 (2011).

28. Dalmadi, A., Gyula, P., Balint, J., Szittya, G. \& Havelda, Z. AGO-unbound cytosolic pool of mature miRNAs in plant cells reveals a novel regulatory step at AGO1 loading. Nucleic Acids Res. 47, 9803-9817 (2019).

29. de Felippes, F. F., Ott, F. \& Weigel, D. Comparative analysis of nonautonomous effects of tasiRNAs and miRNAs in Arabidopsis thaliana. Nucleic Acids Res. 39, 2880-2889 (2011).

30. Morel, J. B. et al. Fertile hypomorphic ARGONAUTE (ago1) mutants impaired in post-transcriptional gene silencing and virus resistance. Plant Cell 14, 629-639 (2002)

31. Miller, J. C., Chezem, W. R. \& Clay, N. K. Ternary WD40 repeat-containing protein complexes: evolution, composition and roles in plant immunity. Front. Plant Sci. 6, 1108 (2015).

32. Xu, C. \& Min, J. Structure and function of WD40 domain proteins. Protein Cell 2, 202-214 (2011).

33. Xie, D. et al. Phase separation of SERRATE drives dicing body assembly and promotes miRNA processing in Arabidopsis. Nat. Cell Biol. 23, 32-39 (2021).

34. Vaucheret, H., Vazquez, F., Crete, P. \& Bartel, D. P. The action of ARGONAUTE1 in the miRNA pathway and its regulation by the miRNA pathway are crucial for plant development. Genes Dev. 18, 1187-1197 (2004).

35. Jia, T. et al. The Arabidopsis MOS4-associated complex promotes MicroRNA biogenesis and precursor messenger RNA splicing. Plant Cell 29, 2626-2643 (2017).

36. $\mathrm{Li}, \mathrm{S}$. et al. MAC3A and MAC3B, two core subunits of the MOS4-associated complex, positively influence miRNA biogenesis. Plant Cell 30, 481-494 (2018).

37. Wang, S. et al. The PROTEIN PHOSPHATASE4 complex promotes transcription and processing of primary microRNAs in Arabidopsis. Plant Cell 31, 486-501 (2019).

38. Laubinger, S. et al. Dual roles of the nuclear cap-binding complex and SERRATE in pre-mRNA splicing and microRNA processing in Arabidopsis thaliana. Proc. Natl Acad. Sci. USA 105, 8795-8800 (2008).

39. Zhan, X. et al. Arabidopsis proline-rich protein important for development and abiotic stress tolerance is involved in microRNA biogenesis. Proc. Natl Acad. Sci. USA 109, 18198-18203 (2012).

40. Zielezinski, A. et al. mirEX 2.0-an integrated environment for expression profiling of plant microRNAs. BMC Plant Biol. 15, 144 (2015)

41. Kim, S. et al. Two cap-binding proteins CBP20 and CBP80 are involved in processing primary MicroRNAs. Plant Cell Physiol. 49, 1634-1644 (2008)

42. Ben Chaabane, S. et al. STA1, an Arabidopsis pre-mRNA processing factor 6 homolog, is a new player involved in miRNA biogenesis. Nucleic Acids Res. 41, 1984-1997 (2013).

43. Ren, G. et al. Regulation of miRNA abundance by RNA binding protein TOUGH in Arabidopsis. Proc. Natl Acad. Sci. USA 109, 12817-12821 (2012).

44. Qiao, Y., Shi, J., Zhai, Y., Hou, Y. \& Ma, W. Phytophthora effector targets a novel component of small RNA pathway in plants to promote infection. Proc. Natl Acad. Sci. USA 112, 5850-5855 (2015).

45. Furumizu, C., Tsukaya, H. \& Komeda, Y. Characterization of EMU, the Arabidopsis homolog of the yeast THO complex member HPR1. RNA 16, 1809-1817 (2010).

46. Francisco-Mangilet, A. G. et al. THO2, a core member of the THO/TREX complex, is required for microRNA production in Arabidopsis. Plant J. 82, 1018-1029 (2015).

47. $\mathrm{Wu}, \mathrm{X}$. et al. A role for the RNA-binding protein MOS2 in microRNA maturation in Arabidopsis. Cell Res. 23, 645-657 (2013).

48. Yu, B. et al. The FHA domain proteins DAWDLE in Arabidopsis and SNIP1 in humans act in small RNA biogenesis. Proc. Natl Acad. Sci. USA 105 10073-10078 (2008).

49. Zhang, S. X., Liu, Y. H. \& Yu, B. PRL1, an RNA-binding protein, positively regulates the accumulation of miRNAs and siRNAs in Arabidopsis. PloS Genetics 10, e1004841 (2014).

50. Iki, T. et al. In vitro assembly of plant RNA-induced silencing complexes facilitated by molecular chaperone HSP90. Mol. Cell 39, 282-291 (2010).

51. Iwasaki, S. et al. Hsc70/Hsp90 chaperone machinery mediates ATP-dependent RISC loading of small RNA duplexes. Mol. Cell 39, 292-299 (2010).
52. Johnston, M., Geoffroy, M. C., Sobala, A., Hay, R. \& Hutvagner, G. HSP90 protein stabilizes unloaded argonaute complexes and microscopic P-bodies in human cells. Mol. Biol. Cell 21, 1462-1469 (2010).

53. Iki, T., Yoshikawa, M., Meshi, T. \& Ishikawa, M. Cyclophilin 40 facilitates HSP90-mediated RISC assembly in plants. EMBO J. 31, 267-278 (2012).

54. Smith, M. R. et al. Cyclophilin 40 is required for microRNA activity in Arabidopsis. Proc. Natl Acad. Sci. USA 106, 5424-5429 (2009).

55. Tomassi, A. H. et al. The intrinsically disordered protein CARP9 bridges HYL1 to AGO1 in the nucleus to promote microRNA activity. Plant Physiol. 184, 316-329 (2020).

56. Murray, M. G. \& Thompson, W. F. Rapid isolation of high molecular weight plant DNA. Nucleic Acids Res. 8, 4321-4325 (1980).

57. Nakamura, S. et al. Gateway binary vectors with the bialaphos resistance gene, bar, as a selection marker for plant transformation. Biosci. Biotechnol. Biochem. 74, 1315-1319 (2010).

58. Clough, S. J. \& Bent, A. F. Floral dip: a simplified method for Agrobacteriummediated transformation of Arabidopsis thaliana. Plant J. 16, 735-743 (1998)

59. Liu, C., Axtell, M. J. \& Fedoroff, N. V. The helicase and RNaseIIIa domains of Arabidopsis Dicer-Likel modulate catalytic parameters during microRNA biogenesis. Plant Physiol. 159, 748-758 (2012).

60. Langmead, B., Trapnell, C., Pop, M. \& Salzberg, S. L. Ultrafast and memoryefficient alignment of short DNA sequences to the human genome. Genome Biol. 10, R25 (2009).

61. Nobuta, K., McCormick, K., Nakano, M. \& Meyers, B. C. Bioinformatics analysis of small RNAs in plants using next generation sequencing technologies. Methods Mol. Biol. 592, 89-106 (2010).

62. Love, M. I., Huber, W. \& Anders, S. Moderated estimation of fold change and dispersion for RNA-seq data with DESeq2. Genome Biol. 15, 550 (2014).

63. Cai, Q. et al. The disease resistance protein $\mathrm{SNC1}$ represses the biogenesis of microRNAs and phased siRNAs. Nat. Commun. 9, 5080 (2018).

64. Jefferson, R. A., Kavanagh, T. A. \& Bevan, M. W. GUS fusions: betaglucuronidase as a sensitive and versatile gene fusion marker in higher plants. EMBO J. 6, 3901-3907 (1987).

65. Li, S. et al. Global co-transcriptional splicing in Arabidopsis and the correlation with splicing regulation in mature RNAs. Mol. Plant 13, 266-277 (2020).

66. Dobin, A. et al. STAR: ultrafast universal RNA-seq aligner. Bioinformatics 29, 15-21 (2013).

67. Trapnell, C. et al. Differential analysis of gene regulation at transcript resolution with RNA-seq. Nat. Biotechnol. 31, 46-4 (2013).

68. Tian, T. et al. agriGO v2.0: a GO analysis toolkit for the agricultural community, 2017 update. Nucleic Acids Res. 45, W122-W129 (2017).

69. Letunic, I. \& Bork, P. Interactive Tree Of Life (iTOL) v5: an online tool for phylogenetic tree display and annotation. Nucleic Acids Res. 49, W293-W296 (2021).

\section{Acknowledgements}

We thank Dr. Detlef Weigel for sharing the pSUC2:amiR-SUL transgenic line. This work was funded by the National Natural Science Foundation of China (31870287 and 31701112), China Postdoctoral Science Foundation (2015M582402), National Institutes of Health (GM129373), and Guangdong Innovation Team Project (2014ZT05S078).

\section{Author contributions}

C.L., Q.C., B.M., and X.C. designed the project and experiments. C.L., Q.C., C.X., F.W. B.Z., and D.C. performed the experiments. C.L, S.L., C.Y., T.L., and L.G. analyzed the data. C.L., Q.C., B.M., and X.C. wrote the paper.

\section{Competing interests}

The authors declare no competing interests.

\section{Additional information}

Supplementary information The online version contains supplementary material available at https://doi.org/10.1038/s41467-022-28872-x.

Correspondence and requests for materials should be addressed to Beixin Mo or Xuemei Chen.

Peer review information Nature Communications thanks Pablo Manavella and Yuichiro Watanabe for their contribution to the peer review of this work.

Reprints and permission information is available at http://www.nature.com/reprints

Publisher's note Springer Nature remains neutral with regard to jurisdictional claims in published maps and institutional affiliations. 
(c) (i) Open Access This article is licensed under a Creative Commons Attribution 4.0 International License, which permits use, sharing, adaptation, distribution and reproduction in any medium or format, as long as you give appropriate credit to the original author(s) and the source, provide a link to the Creative Commons license, and indicate if changes were made. The images or other third party material in this article are included in the article's Creative Commons license, unless indicated otherwise in a credit line to the material. If material is not included in the article's Creative Commons license and your intended use is not permitted by statutory regulation or exceeds the permitted use, you will need to obtain permission directly from the copyright holder. To view a copy of this license, visit http://creativecommons.org/ licenses/by/4.0/.

(C) The Author(s) 2022 